\title{
Post-AGB stars as testbeds of nucleosynthesis in AGB stars
}

\author{
G. Stasińska ${ }^{1}$, R. Szczerba ${ }^{2}$, M. Schmidt ${ }^{2}$, and N. Siódmiak ${ }^{2}$ \\ ${ }^{1}$ LUTH, Observatoire de Meudon, 5 Place Jules Janssen, 92195 Meudon Cedex, France \\ e-mail: grazyna.stasinska@obspm.fr \\ 2 N. Copernicus Astronomical Center, Rabiańska 8, 87-100 Toruń, Poland
}

Received 2 June 2005 / Accepted 30 December 2005

\begin{abstract}
We construct a data base of 125 post-AGB objects (including $\mathrm{R} \mathrm{CrB}$ and extreme helium stars) with published photospheric parameters (effective temperature and gravity) and chemical composition. We estimate the masses of the post-AGB stars by comparing their position in the $\left(\log T_{\text {eff }}, \log g\right)$ plane with theoretical evolutionary tracks of different masses. We construct various diagrams, with the aim of finding clues to AGB nucleosynthesis. This is the first time that a large sample of post-AGB stars has been used in a systematic way for such a purpose and we argue that, in several respects, post-AGB stars should be more powerful than planetary nebulae to test AGB nucleosynthesis. Our main findings are that: the vast majority of objects which do not show evidence of $\mathrm{N}$ production from primary $\mathrm{C}$ have a low stellar mass $\left(M_{\star}<0.56 M_{\odot}\right)$; there is no evidence that objects which did not experience 3rd dredge-up have a different stellar mass distribution than objects that did; there is clear evidence that 3rd dredge-up is more efficient at low metallicity. The sample of known post-AGB stars is likely to increase significantly in the near future thanks to the ASTRO-F and follow-up observations, making these objects even more promising as testbeds for AGB nucleosynthesis.
\end{abstract}

Key words. stars: AGB and post AGB - stars: abundances - stars: evolution - nuclear reactions, nucleosynthesis, abundances

\section{Introduction}

The asymptotic giant branch (AGB) phase, a late stage in the evolution of low and intermediate mass stars, is quite complex. During this phase, various nuclear processes are at work in different zones of the star, and a variety of mixing mechanisms take place (see e.g. Charbonnel 2002; or Lattanzio 2002 for short reviews). Understanding this phase is important, since the elements manufactured during the AGB contribute significantly to the chemical composition of galaxies.

The first models to give some predictions on the stellar yields from AGB stars are those by Iben \& Truran (1978) and Renzini \& Voli (1981). Unfortunately, even nowadays, the state of stellar physics does not allow one to construct models entirely from first principles, and some quantities have to be set as free parameters. The next generation of AGB models (Groenewegen \& de Jong 1993; Marigo et al. 1996) adjusted those free parameters (essentially mass loss rate and mixing length) to reproduce a few observational constraints on stellar populations. Further models have been constructed since then (e.g. by Forestini \& Charbonnel 1997; Boothroyd \& Sackmann 1999; Marigo 2001; Izzard et al. 2004). All those models are so-called synthetic models in that, for the thermal pulse phase, they use analytical expressions to extrapolate certain quantities obtained from full evolutionary calculations up to the planetary nebula ejection. With the availability of fast computers, it is now possible to compute complete AGB models (Karakas et al. 2002; Herwig 2004) that follow all the pulses in detail. Testing model predictions before using them in chemical evolution models of galaxies is vital, especially because even full evolutionary calculations are computationally difficult and still imply some ad hoc parameters (mixing length, mass loss rates etc.). Indeed, as shown recently by Ventura \& D'Antona (2005a,b), the predicted yields of intermediate mass stars depend strongly on the treatment adopted for convection and mass loss and on the nuclear reaction cross-sections.

As mentioned before, synthetic models published since 1993 reproduce some observables (e.g. the luminosity functions of carbon-stars and of lithium-rich stars) by construction. However, additional tests are needed and are crucial to constrain AGB models. The analysis of the chemical composition of planetary nebulae can provide some tests. Such an approach has been adopted recently by Marigo et al. (2003) using a data base of 10 planetary nebulae (PNe) observed in a wide spectral range, from the far infrared to the ultraviolet. Previously, the chemical composition of planetary nebulae samples had been used in a more empirical way to test AGB nucleosynthesis and get an insight into the relation between planetary nebulae, the final products of low and intermediate mass stars and their progenitors. For example, Peimbert (1978) defined 
a category of planetary nebulae, called type I PNe, as objects having $\mathrm{He} / \mathrm{H}>0.125$ and $\mathrm{N} / \mathrm{O}>0.5$. Those $\mathrm{PNe}$ were interpreted as being born from stars that experienced the second dredge-up. While the designation "type I PNe" stayed in the literature, its definition changed several times (see Stasińska 2004). Kingsburgh \& Barlow (1994) compared the nebular $\mathrm{N} / \mathrm{H}$ ratio to the solar $(\mathrm{C}+\mathrm{N}) / \mathrm{H}$ ratio to find out which planetary nebulae exhibit $\mathrm{N}$ produced from primary $\mathrm{C}$. They also identified planetary nebulae whose progenitors had experienced 3 rd dredge-up, bringing to the surface carbon produced by the triple- $\alpha$ reaction. To this end, they constructed a $(\mathrm{C}+\mathrm{N}+\mathrm{O}) / \mathrm{H}$ vs. $\mathrm{C} / \mathrm{H}$ diagram. The $\mathrm{N} / \mathrm{O}$ vs. $\mathrm{O} / \mathrm{H}$ diagram has been interpreted (e.g. Henry 1990) as indicating that in some objects $\mathrm{N}$ is produced at the expense of $\mathrm{O}$ (during $\mathrm{ON}$ cycling). However, this diagram, depending on authors and samples, does not always lead to such a straightforward interpretation (Kingsburgh \& Barlow 1994; Leisy \& Dennefeld 1996). Henry et al. (2000) have plotted PN abundances of $\mathrm{C}$ and $\mathrm{N}$ as a function of progenitor mass (estimated from the stellar remnant masses by Górny et al. 1997 and Stasińska et al. 1997 and converted to progenitor masses using an initial-final mass relation), and compared this with the predictions from synthetic AGB models. This was the first attempt to compare PNe abundances directly with the results of AGB model computations for a given initial mass and it was not very conclusive.

There is another category of objects that may serve for tests of AGB models. These are post-AGB stars, i.e. stars that have already ejected their envelope but are not yet hot enough to ionize it and produce a planetary nebula. Such stars have several advantages with respect to PNe and constitute an excellent complement for testing AGB nucleosynthesis. The main advantage is that the stellar mass can be obtained directly from the observed stellar spectrum by fitting a model atmosphere that allows one to derive the stellar effective temperature, $T_{\text {eff }}$, and gravity $g$ (this can also be done for PNe nuclei, but as the stars are hotter and buried in the ionized gas, this is more difficult). The abundances of quite a variety of elements $(\mathrm{He}, \mathrm{Li}$, C, N, O, Na, Mg, Al, Si, S, Ca, Sc, Ti, Fe, Ni, Zn ...) can be obtained from a stellar atmosphere analysis. For carbon, a particularly important element in AGB evolution, the uncertainty in the estimated abundance is of the same order as for the other elements. This is not the case in planetary nebulae, where no strong carbon line exists in the optical, implying that the carbon abundance determination is subject to a higher uncertainty than the oxygen or the nitrogen abundance determination. Finally, post-AGB stars are expected to extend to smaller masses than the nuclei of planetary nebulae. Indeed, planetary nebulae with central star masses lower than $0.55 M_{\odot}$ do not exist since the nebular gas has dissipated in the interstellar medium long before the star has become hot enough to ionize it. On the other hand, there is no such limitation for post-AGB stars.

This paper presents the first attempt to use a large sample of post-AGB stars to test AGB nucleosynthesis. In Sect. 2, we describe the constitution of our sample. In Sect. 3 we show some empirical diagrams and propose simple interpretations. In Sect. 4 we summarize the main findings and outline some prospects.

\section{The optical sample of post-AGB stars}

\subsection{General presentation}

As mentioned above, the post-AGB phase starts when the star has expelled its envelope and left the AGB branch, and is then moving to the left in the H-R diagram. The end of AGB is characterized by strong mass loss, which (sometimes) can reach values of $10^{-4} M_{\odot} \mathrm{yr}^{-1}$. For stars that have experienced intense mass loss on the AGB, due to the large dust opacity in the envelope, the post-AGB star is first seen through its infrared emission due to reprocessing of the stellar radiation by the circumstellar dust grains. It is only when the envelope has sufficiently expanded and become optically thin that such stars start being optically visible. In this paper, we are interested in optically visible post-AGB stars. We used the present version (Szczerba et al., in preparation) of our catalogue of postAGB candidates (Szczerba et al. 2001), which now contains about 330 objects. For all the sources from this catalogue we have searched the available literature for stellar parameters and chemical composition.

We then estimated the stellar masses, $M_{\star}$, by comparison with theoretical evolutionary paths in the $\left(\log T_{\text {eff }}, \log g\right)$ plane. The theoretical paths we used were interpolated by Górny et al. (1997) from the post-AGB models of Schönberner (1983) and Blöcker (1995). The uncertainties in $\log T_{\text {eff }}$ and $\log g$ induce an uncertainty in the derived $M_{\star}$. The main source of uncertainties is the low accuracy of the determinations of surface gravity, especially in the case of cool post-AGB stars ( $T_{\text {eff }}$ below $10000 \mathrm{~K}$ ). Since the relation between $\log g$ and stellar mass on the post-AGB tracks is highly nonlinear, for each object we determined the mass $M_{\star \text { min }}$ corresponding to $\left(\log T_{\text {eff }}-\Delta\left(\log T_{\text {eff }}\right), \log g-\Delta(\log g)\right)$ and the mass $M_{\star \max }$ corresponding to $\left(\log T_{\text {eff }}+\Delta\left(\log T_{\text {eff }}\right), \log g+\Delta(l \log g)\right)$. Since the derived stellar mass is a decreasing function of both $T_{\text {eff }}$ and $\log g$, the values of $M_{\star \min }$ and $M_{\star \max }$ should define a conservative stellar mass interval ${ }^{1}$. However, one should keep in mind that many post-AGB stars are pulsating stars (Gautschy \& Saio 1996), while such pulses are not reproduced by the evolutionary models used to derive the stellar masses. In addition, the pulsating nature of the atmosphere may introduce errors in the abundance determinations, which are done using static atmosphere models. A further source of uncertainty in the determination of masses comes, of course, from the model tracks themselves. All this implies that the masses of postAGB stars are quite uncertain and model-dependent. In spite of this, we believe that the method provides useful information. It can be improved on in the future, as better spectra become available for spectroscopic analysis and as progress in understanding the physics of post-AGB stars is made. In particular, when complete grids with different metallicities become available, it should be possible to account for the metallicity in the derivation of the stellar masses. For the moment, as far as one can judge from several tracks computed by Vassiliadis \& Wood (1994) at different metallicities, the effect of metallicity in mass

\footnotetext{
1 The available model grid allows us to determine masses only within the range $\left(0.55-0.94 M_{\odot}\right)$.
} 
derivation would be completely dominated by the large error on $\log g$.

The details of our search and the above estimates of the stellar masses are presented in Table 1. The objects are grouped into several subtypes: RV Tau stars (29 objects), suspected RV Tau stars (6 objects from Maas et al. 2005), R CrB stars (19 objects), extreme helium stars (15 objects) and all the remaining post-AGB stars (56 objects).

The RV Tau stars are highly luminous variable stars characterized by light-curves with alternate deep and shallow minima, periods between 30 and 150 days, and F, G or K spectral types (see e.g. Preston et al. 1963). They have been identified as postAGB stars by Jura (1986), who showed that their IRAS fluxes indicate that they have just left a phase of very rapid mass loss.

The R CrB stars (already known for more than 200 years!) are rare $\mathrm{H}$-deficient and $\mathrm{C}$-rich supergiants that undergo irregular declines of up to 8 mag when dust forms in clumps along the line of sight (see e.g Clayton 1996, for a review). The extreme $\mathrm{H}$-deficiency of the $\mathrm{R} \mathrm{CrB}$ stars suggests that some mechanism removed the entire H-rich stellar envelope. There are two major models which explain their origin: a merger scenario (Webbink 1984; Iben et al. 1986) or the final helium shell flash scenario (Fujimoto 1977; Renzini 1979). There is still no consensus about which scenario is valid (none of them can explain all the observed properties). Only the second scenario implies a post-AGB nature. We have included these stars in Table 1, but we do not consider them as bona-fide post-AGB stars. Extreme helium stars, which could be evolutionarily connected to R CrB stars (see e.g. Pandey et al. 2001), are also included in our table and discussed together with $\mathrm{R} \mathrm{CrB}$ stars.

In each subtype, the objects in Table 1 are ordered by galactic coordinates $l$ and $b$. For some sources, there are several entries and in Sect. 2.2 we briefly discuss the preferred determinations - usually, they are based on higher quality spectroscopic material. The columns contain the following data: (1) the object number; (2) the object coordinates $l, b$; (3) the IRAS name; (4) the HD number; (5) other name (either the usual name or the designation in one of the following catalogues (chosen in this order: General Catalogue of Variable Stars, LS, BD, SAO, and CD catalogues); (6) the effective tempearture; (7) the error attributed to $T_{\mathrm{eff}} ;(8)$ the logarithm of the surface gravity in $\mathrm{cm} \mathrm{s}^{-2}$; (9) the error attributed to $\log g$; (10) references and notes for the collected stellar parameters (explanations for the abbreviations used and for notes are given at the end of the table); (11) $M_{\star}$ in units of solar mass; (12) $M_{\star \min }$ and (13) $M_{\star \max }$.

Table 2 is ordered in the same way as Table 1 . Its columns contain: (1) the object number; (2) the object coordinates $l$, $b$; (3) - (8) the abundances of $\mathrm{C}, \mathrm{N}, \mathrm{O}, \mathrm{S}, \mathrm{Fe}$, and $\mathrm{Zn}$, expressed as $\epsilon(\mathrm{X})=12+\log (\mathrm{X} / \mathrm{H})$ where $\mathrm{X} / \mathrm{H}$ is the abundance of element $\mathrm{X}$ in number relative to $\mathrm{H}$. In the case of $\mathrm{R} \mathrm{CrB}$ and extreme helium stars, the abundances are listed as $\epsilon(\mathrm{X})=$ $12.15+\log (\mathrm{X} / N)$, where $\mathrm{X} / N$ is the ratio of number density of element $\mathrm{X}$ to the total number density of nucleons $N$. Note that the second definition is more general than the earlier one and both are consistent if the abundance of helium amounts to $\epsilon(\mathrm{He})=11$, a condition which is fulfilled with good accuracy in the remaining stars (see Asplund et al. 2000; and
Pandeyet al. 2001). The sources for the abundances are the same as given in Col. 8 of Table 1. Overall, the typical uncertainty in elemental abundances is about $0.2-0.3$ dex. However, the uncertainty in abundance ratios of heavy elements is smaller, since many sources of errors affect the derived abundances in a similar way. One exception is the case of oxygen, if its abundance has been determined from the O I 7771-5 triplet. It is well known that this triplet gives enhanced abundances, if non-LTE effects are not taken into account, and that the O I 7771-5 vs. [O I] discrepancy is higher for low metallicity (Takeda 2003). In most objects listed in Table 2, it appears that the use of this triplet was avoided.

\subsection{Notes on individual objects and discussion of abundance uncertainties}

For several sources we found more than one reference with stellar parameters and chemical composition determined. Below we present arguments for the preferred source of information. The object number corresponds to the number given in Col. 1 of Tables 1 and 2.

Object 1: IRAS 18384-2800. The atmospheric parameters and chemical abundances have been analyzed recently by AFGM01 and RvW01. The same atmospheric parameters were derived in both analyses. The spectra obtained by RvW01 have apparently much higher S/N-ratio than those of AFGM01, and the chemical analysis of RvW01 is based on a higher number of lines, so the results of RvW01 were chosen for further analysis.

Object 3: IRAS 17279-1119. This star has been analyzed by AFGM01 and VW97. Both analyses gave similar value for $T_{\text {eff }}$ but rather different values for the surface gravity. Since the determination of AFGM01 was based on a single spectrum, while the vW97 determination was based on several spectra at different photometric phases, it is likely that more consistent atmospheric parameters and chemical composition are obtained in the AFGM01 paper.

Object 5: IRAS 19500-1709. Both analyses by vWR00 and vWWW96 give similar atmospheric parameters. The vWR00 paper is based on a higher $\mathrm{S} / \mathrm{N}$ and a broader spectral coverage and was chosen for the subsequent analysis.

Object 6: IRAS 19590-1249. We adopted the RDM03 values. The analysis of RDM03 is based on higher quality material and is based on fully blanketed non-LTE atmospheric models that should guarantee more accurate values for $T_{\text {eff }}$ and $\log g$.

Object 8: PHL 1580. KL86 do not give abundances, so we had to rely on those given by CDK91.

Object 10: IRAS 19114+0002. The analysis of RH99 is based on higher quality material than in ZKP96. As a consequence the microturbulence derived by RH99 $\left(5.25 \mathrm{~km} \mathrm{~s}^{-1}\right)$ is much lower than the supersonic value found by ZKP99 $\left(8 \mathrm{~km} \mathrm{~s}^{-1}\right)$. Since TPJ00 give a really unusual result we selected the data from RH99.

Object 12: PG 1704+222. The analysis of MH98 is based on better quality spectra than the preliminary results of CTM93, and their results were adopted.

Object 14: IRAS 18062+2410. There are emission lines visible in the spectrum. The four papers containing a determination 
Table 1. List of post-AGB objects with stellar parameters determined from model atmosphere techniques.

\begin{tabular}{|c|c|c|c|c|c|c|c|c|c|c|c|}
\hline $\begin{array}{l}\text { No. } \\
\text { (1) }\end{array}$ & $\begin{array}{l}l \quad b \\
(2)\end{array}$ & $\begin{array}{l}\text { IRAS } \\
\text { (3) }\end{array}$ & $\begin{array}{l}\text { HD } \\
\text { (4) }\end{array}$ & $\begin{array}{l}\text { Other name } \\
\text { (5) }\end{array}$ & $\begin{array}{l}T_{\text {eff }} \\
\text { (6) }\end{array}$ & $\begin{array}{c}\text { Err. } \log g \\
\text { (7) (8) }\end{array}$ & $\begin{array}{l}\text { Err. } \\
(9)\end{array}$ & $\begin{array}{l}\text { Notes } \\
(10)\end{array}$ & $\begin{array}{r}M_{\star} \\
(11)\end{array}$ & $\begin{array}{r}M_{\star \min } \\
(12)\end{array}$ & $\begin{array}{r}M_{\star \max } \\
\quad(13)\end{array}$ \\
\hline \multirow[t]{2}{*}{1} & $006.72-10.37$ & $18384-2800$ & 172481 & V4728 Sgr & 7250 & 2001.5 & 0.25 & AFGM01 & $<0.550$ & $<0.550<$ & $<0.550$ \\
\hline & & & & & 7250 & $250 \quad 1.5$ & 0.5 & RvW01 & $<0.550$ & $<0.550$ & 0.554 \\
\hline 2 & $007.96+26.71$ & F16277-0724 & 148743 & BD-07 4305 & 7200 & $500 \quad 0.5$ & 0.3 & LBL90 $^{1)}$ & 0.828 & $0.627>$ & $>0.940$ \\
\hline \multirow[t]{2}{*}{3} & $013.23+12.17$ & $17279-1119$ & 158616 & V340 Ser & 7550 & $150 \quad 0.75$ & 0.25 & vW972) & 0.632 & 0.576 & 0.874 \\
\hline & & & & & 7300 & 2001.5 & 0.25 & AFGM01 & $<0.550$ & $<0.550<$ & $<0.550$ \\
\hline 4 & $016.45-50.43$ & & & BPS CS 29493-0046 & 20000 & 30003.0 & 0.3 & KDK97 & 0.550 & $<0.550$ & 0.550 \\
\hline \multirow[t]{2}{*}{5} & $023.98-21.04$ & $19500-1709$ & 187885 & V5112 Sgr & 8000 & 2501.0 & 0.5 & vWR00 & 0.599 & $<0.550$ & 0.933 \\
\hline & & & & & 7850 & $150 \quad 0.75$ & 0.25 & vWWW96 & 0.673 & 0.599 & 0.926 \\
\hline \multirow[t]{2}{*}{6} & $029.18-21.26$ & $19590-1249$ & & LS IV -12 111 & 20500 & $500 \quad 2.35$ & 0.2 & $\mathrm{RDM}^{3)}$ & 0.757 & 0.625 & 0.931 \\
\hline & & & & & 23750 & 10002.7 & 0.2 & MCD92 & 0.649 & 0.612 & 0.826 \\
\hline 7 & $030.60-21.53$ & $20023-1144$ & 190390 & V1401 Aql & 6600 & 5001.6 & 0.3 & LBL90 $^{1)}$ & $<0.550$ & $<0.550<$ & $<0.550$ \\
\hline \multirow[t]{2}{*}{8} & $031.33-43.48$ & & & PHL 1580 & 24000 & 10003.6 & 0.2 & CDK91 & $<0.550$ & $<0.550<$ & $<0.550$ \\
\hline & & & & & 21500 & 15003.0 & 0.35 & KL86 & 0.552 & $<0.550$ & 0.578 \\
\hline 9 & $033.16-48.12$ & & & PHL 174 & 18000 & 10002.7 & 0.2 & CDK91 & 0.552 & 0.550 & 0.556 \\
\hline \multirow[t]{3}{*}{10} & $035.62-04.96$ & $19114+0002$ & 179821 & V1427 Aql & 6750 & 1500.5 & 0.5 & RH99 & 0.660 & $0.553>$ & $>0.940$ \\
\hline & & & & & 6800 & $250 \quad 1.3$ & 0.5 & $\mathrm{ZKI}$ & $<0.550$ & $<0.550$ & 0.559 \\
\hline & & & & & 5660 & $100-1.0$ & 0.5 & TPJ00 & $>0.940$ & $>0.940>$ & $>0.940$ \\
\hline 11 & $040.51-10.09$ & $19386+0155$ & & V1648 Aql & 6800 & 1001.4 & 0.2 & PLM04 & $<0.550$ & $<0.550<$ & $<0.550$ \\
\hline \multirow[t]{2}{*}{12} & $043.06+32.36$ & & & PG $1704+222$ & 20500 & 10003.0 & 0.2 & СТМ93 & 0.551 & $<0.550$ & 0.554 \\
\hline & & & & & 17600 & 4002.7 & 0.1 & MH984) & 0.551 & 0.550 & 0.553 \\
\hline 13 & $043.23-57.13$ & $22327-1731$ & 213985 & HM Aqr & 8200 & 1.5 & & vW95 & $<0.550$ & & \\
\hline \multirow[t]{4}{*}{14} & $050.67+19.79$ & $18062+2410$ & 341617 & V886 Her & 23000 & 10002.6 & 0.2 & MRK02 & 0.692 & 0.621 & 0.868 \\
\hline & & & & & 23000 & 2003.0 & 0.25 & AFGM01 & 0.558 & 0.550 & 0.614 \\
\hline & & & & & 22000 & 2003.0 & 0.5 & PGSO0 & 0.554 & $<0.550$ & 0.697 \\
\hline & & & & & 20750 & $500 \quad 2.35$ & 0.2 & $\mathrm{RDM}^{33)}$ & 0.813 & $0.631>$ & $>0.940$ \\
\hline 15 & $051.43+23.19$ & $17534+2603$ & 163506 & 89 Her & 6550 & 5000.6 & 0.3 & LBL90 ${ }^{1)}$ & 0.611 & 0.561 & 0.670 \\
\hline 16 & $052.73+50.79$ & & & $\mathrm{BD}+332642$ & 20200 & $500 \quad 2.9$ & 0.1 & NHK94 & 0.552 & 0.551 & 0.554 \\
\hline 17 & $053.84+20.18$ & $18095+2704$ & & V887 Her & 6600 & $300 \quad 1.05$ & 0.5 & K95 $5^{5)}$ & 0.550 & $<0.550$ & 0.607 \\
\hline 18 & $066.18+18.58$ & & 172324 & V534 Lyr & 11250 & 2002.5 & 0.25 & AFGM016) & $<0.550$ & $<0.550<$ & $<0.550$ \\
\hline \multirow[t]{2}{*}{19} & $067.16+02.73$ & $19475+3119$ & 331319 & LS II +319 & 7250 & $100 \quad 0.5$ & 0.3 & КРТ02 & 0.841 & $0.611>$ & $>0.940$ \\
\hline & & & & & 7750 & 2001.0 & 0.25 & AFGM01 & 0.578 & 0.553 & 0.632 \\
\hline \multirow[t]{2}{*}{20} & $077.13+30.87$ & $17436+5003$ & 161796 & V814 Her & 6600 & $\begin{array}{lll}500 & 0.25\end{array}$ & 0.3 & LBL90 $^{1)}$ & 0.915 & $0.698>$ & $>0.940$ \\
\hline & & & & & 7100 & 1000.5 & 0.3 & & 0.785 & $0.604>$ & $>0.940$ \\
\hline 21 & $080.17-06.50$ & & & Egg & 6500 & 2000.0 & 0.3 & & $>0.940$ & $0.897>$ & $>0.940$ \\
\hline \multirow[t]{2}{*}{22} & $096.75-11.56$ & $22223+4327$ & & $\mathrm{BD}+424388$ & 6500 & 2501.0 & 0.5 & vWR00 & 0.551 & $<0.550$ & 0.614 \\
\hline & & & & & 6500 & 3501.0 & 0.5 & DvWW98 & 0.551 & $<0.550$ & 0.609 \\
\hline 23 & $098.41-16.73$ & & & $\mathrm{BD}+394926$ & 7500 & 0.5 & & K73 & 0.894 & & \\
\hline \multirow[t]{2}{*}{24} & $103.35-02.52$ & $22272+5435$ & 235858 & V354 Lac & 5750 & $150 \quad 0.5$ & 0.5 & RLG02 & 0.574 & & 0.888 \\
\hline & & & & & 5600 & $250 \quad 0.5$ & 0.5 & ZKP95 & 0.561 & $<0.550$ & 0.805 \\
\hline \multirow[t]{2}{*}{25} & $113.86+00.59$ & $23304+6147$ & & & 6750 & $250 \quad 0.5$ & 0.5 & vWR00 & 0.660 & $0.554>$ & $>0.940$ \\
\hline & & & & & 5900 & 2000.0 & 0.5 & KSP00a & $>0.940$ & $0.606>$ & $>0.940$ \\
\hline 26 & $123.57+16.59$ & $01005+7910$ & & & 21000 & 5003.0 & 0.3 & KYM02 & 0.551 & $<0.550$ & 0.576 \\
\hline 27 & $133.73+01.50$ & $Z 02229+6208$ & & & 5500 & 2500.5 & 0.25 & RBH99 & 0.558 & 0.551 & 0.604 \\
\hline 28 & $161.98+19.59$ & $06338+5333$ & 46703 & V382 Aur & 6000 & $\begin{array}{ll}150 & 0.4\end{array}$ & 0.3 & LB84 & 0.620 & 0.558 & 0.867 \\
\hline \multirow[t]{3}{*}{29} & $166.24-09.05$ & $04296+3429$ & & & 7000 & 2501.0 & 0.5 & vWR00 & 0.554 & $<0.550$ & 0.660 \\
\hline & & & & & 6300 & $250 \quad 0.0$ & 0.2 & KSP99 & $>0.940$ & $0.938>$ & $>0.940$ \\
\hline & & & & & 7000 & 3501.0 & 0.3 & DvWW98 & 0.554 & $<0.550$ & 0.591 \\
\hline 30 & $172.95-05.50$ & & & Barnard 29 & 20000 & 10003.0 & 0.1 & CDK94 & 0.550 & 0.550 & 0.550 \\
\hline 31 & $173.86-82.41$ & & & BPS CS 22946-0005 & 20000 & 30002.7 & 0.3 & KDK97 & 0.565 & 0.558 & 0.571 \\
\hline 32 & $188.86-14.29$ & $05113+1347$ & & & 5250 & 1500.25 & 0.5 & RLG02 & 0.604 & $0.550>$ & $>0.940$ \\
\hline 33 & $196.19-12.14$ & $05341+0852$ & & & 6500 & 2501.0 & 0.5 & vWR00 & 0.551 & $<0.550$ & 0.614 \\
\hline 34 & $204.67+07.57$ & $07008+1050$ & 52961 & PS Gem & 6000 & $500 \quad 0.5$ & 0.5 & WvWB91 & 0.599 & 0.551 & 0.860 \\
\hline \multirow[t]{3}{*}{35} & $206.75+09.99$ & $07134+1005$ & 56126 & LS VI +10 15 & 7250 & $250 \quad 0.5$ & 0.5 & HR03 & 0.841 & $0.563>$ & $>0.940$ \\
\hline & & & & & 7250 & 2500.5 & 0.5 & vWR00 & 0.841 & $0.563>$ & $>0.940$ \\
\hline & & & & & 7000 & $300 \quad 0.1$ & 0.5 & K95 & $>0.940$ & $0.697>$ & $>0.940$ \\
\hline 36 & $208.93+17.07$ & $07430+1115$ & & & 6000 & 2501.0 & 0.25 & RBH99 & $<0.550$ & 0.540 & 0.551 \\
\hline
\end{tabular}


Table 1. continued.

\begin{tabular}{|c|c|c|c|c|c|c|c|c|c|c|c|c|}
\hline \multicolumn{2}{|c|}{$\begin{array}{l}\text { No. } l \quad b \\
\text { (1) (2) }\end{array}$} & $\begin{array}{l}\text { IRAS } \\
(3)\end{array}$ & $\begin{array}{l}\text { HD } \\
(4)\end{array}$ & $\begin{array}{l}\text { Other name } \\
\text { (5) }\end{array}$ & $\begin{array}{r}T_{\text {eff }} \\
(6) \\
\end{array}$ & \multicolumn{2}{|c|}{$\begin{array}{c}\text { Err. } \log g \\
\text { (7) (8) }\end{array}$} & $\begin{array}{l}\text { Err. } \\
(9)\end{array}$ & $\begin{array}{l}\text { Notes } \\
(10)\end{array}$ & $\begin{array}{r}M_{\star} \\
(11)\end{array}$ & $\begin{array}{r}M_{\star \min } \\
(12) \\
\end{array}$ & $\begin{array}{r}M_{\star \max } \\
(13) \\
\end{array}$ \\
\hline 37 & $215.44-00.13$ & $06530-0213$ & & & 6900 & 250 & 1.0 & 0.5 & HR03 & 0.553 & 0.540 & 0.643 \\
\hline & & & & & 7250 & 250 & 1.0 & 0.5 & RvWG04 & $0.557<$ & $<0.550$ & 0.722 \\
\hline 38 & $218.97-11.76$ & $06176-1036$ & 44179 & Red Rectangle & 7500 & & 0.8 & & WvWTW92 & 0.617 & & \\
\hline 39 & $236.57-05.37$ & $07140-2321$ & & SAO 173329 & 6750 & 250 & 1.25 & 0.25 & vW97²) & $<0.550<$ & $<0.550$ & 0.551 \\
\hline 40 & $260.83-05.07$ & $08143-4406$ & & & 7150 & 100 & 1.35 & 0.15 & RvWG047) & $<0.550<$ & $<0.550$ & 0.550 \\
\hline 41 & $264.55+72.47$ & & 105262 & $\mathrm{BD}+132491$ & 9250 & 250 & 1.8 & 0.25 & RPS96 & $<0.550<$ & $<0.550$ & 0.551 \\
\hline 42 & $266.85+22.93$ & $10158-2844$ & 89353 & AG Ant & 7600 & 400 & 1.05 & 0.1 & WvWB91 & 0.559 & 0.558 & 0.559 \\
\hline 43 & $269.97-34.08$ & & & CPD-61 455 & 25000 & 1000 & 3.6 & 0.2 & HDK96 & $<0.550<$ & $<0.550$ & $<0.550$ \\
\hline 44 & $290.54-01.95$ & $11000-6153$ & 95767 & LS 2105 & 7300 & 300 & 1.5 & 0.25 & vW972) & $<0.550<$ & $<0.550$ & $<0.550$ \\
\hline 45 & $293.03+05.94$ & $11385-5517$ & 101584 & V885 Cen & 8500 & 500 & 1.5 & 0.5 & SPG99 & $0.550<$ & $<0.550$ & 0.599 \\
\hline 46 & $295.48+29.87$ & & 107369 & SAO 203367 & 7600 & 400 & 1.5 & 0.25 & vW972) & $<0.550<$ & $<0.550$ & 0.550 \\
\hline 47 & $298.25+15.48$ & $12222-4652$ & 108015 & SAO 223420 & 6800 & 200 & 1.25 & 0.25 & vW972) & $<0.550<$ & $<0.550$ & 0.551 \\
\hline 48 & $298.30+08.67$ & $12175-5338$ & & V1024 Cen & 7350 & 150 & 0.75 & 0.25 & vW972) & 0.620 & 0.563 & 0.828 \\
\hline 49 & $299.02-43.77$ & & & LB 3219 & 21250 & 1000 & 2.8 & 0.2 & MCD92 & 0.567 & 0.555 & 0.603 \\
\hline \multirow[t]{2}{*}{50} & $304.34+36.40$ & $12538-2611$ & 112374 & LN Hya & 6000 & 200 & 1.0 & 0.25 & GAFP97 & $<0.550<$ & $<0.550$ & 0.552 \\
\hline & & & & & 6000 & 275 & 0.6 & 0.3 & LLB83 & 0.568 & 0.551 & 0.624 \\
\hline 51 & $309.07+15.18$ & & 116745 & Fehrenbach's star & 6950 & 75 & 1.15 & 0.1 & GW92 & $0.550<$ & $<0.550$ & 0.552 \\
\hline \multirow[t]{2}{*}{52} & $317.11+53.11$ & & & BPS CS 22877-0023 & 20000 & 3000 & 3.0 & 0.3 & KDK97 & $0.550<$ & $<0.550$ & 0.550 \\
\hline & & & & & 16400 & 700 & 2.5 & 0.15 & MH984) & 0.553 & 0.551 & 0.557 \\
\hline 53 & $325.04+08.65$ & $15039-4806$ & 133656 & LS 3309 & 8000 & 200 & 1.25 & 0.25 & vWOT96 ${ }^{8)}$ & $0.554<$ & $<0.550$ & 0.582 \\
\hline \multirow[t]{2}{*}{54} & $326.77-07.49$ & $16206-5956$ & & LS 3591 & 11200 & 1000 & 2.3 & 1.0 & GP03 & $<0.550<$ & $<0.550$ & 0.624 \\
\hline & & & & & 8500 & 200 & 0.9 & 0.2 & VSL98 & 0.660 & 0.609 & 0.874 \\
\hline 55 & $330.64-03.67$ & & & LS 3593 & 9300 & 200 & 1.7 & 0.2 & VSL98 & $<0.550<$ & $<0.550$ & 0.553 \\
\hline 56 & $345.58-07.30$ & & & [DSH2001] 279-19 & 24000 & 1000 & 3.3 & 0.2 & MDS04 & $0.550<$ & $<0.550$ & 0.553 \\
\hline & Tau stars & & & & & & & & & & & \\
\hline 57 & $020.72+17.57$ & & & V453 Oph & 5800 & 200 & 0.75 & 0.25 & GLG98 & $0.552<$ & $<0.550$ & 0.561 \\
\hline & & & & & 6250 & & 1.5 & & RDvW04 & $<0.550$ & & \\
\hline 58 & $021.48+28.63$ & & & TT Oph & 4800 & 200 & 0.5 & 0.25 & GLG00 & 0.550 & $<0.550$ & 0.555 \\
\hline 59 & $026.52-05.42$ & $18564-0814$ & & AD Aql & 6300 & 150 & 1.25 & 0.15 & GLG98 & $<0.550<$ & $<0.550$ & $<0.550$ \\
\hline 60 & $044.10-61.56$ & & 216457 & DS Aqr & 6500 & 200 & 1.0 & 0.25 & GLG00 & $0.551<$ & $<0.550$ & 0.558 \\
\hline & & & & & 6500 & 200 & 2.0 & 0.25 & GLG98 & $<0.550<$ & $<0.550$ & $<0.550$ \\
\hline & & & & & 5750 & & 0.5 & & RDvW04 & 0.574 & & \\
\hline 61 & $050.49+14.24$ & $18281+2149$ & 170756 & AC Her & 5900 & 150 & 1.13 & 0.15 & GLG98 ${ }^{6}$ & $<0.550<$ & $<0.550$ & $<0.550$ \\
\hline 62 & $057.53-09.75$ & $20117+1634$ & 192388 & R Sge & 5000 & 200 & -0.25 & 0.2 & GLG97a 9) & 0.926 & 0.806 & $>0.940$ \\
\hline 63 & $058.44-07.46$ & $20056+1834$ & & QY Sge & 5850 & 200 & 0.7 & 0.25 & RGL02 & 0.554 & $<0.550$ & 0.579 \\
\hline 64 & $060.73+06.94$ & $19163+2745$ & & EP Lyr & 6200 & 200 & 1.4 & 0.2 & GLG97a9) $^{9}$ & $<0.550<$ & $<0.550$ & $<0.550$ \\
\hline 65 & $076.75-11.78$ & & & V360 Cyg & 5275 & 200 & 1.38 & 0.25 & GLG986) & $<0.550<$ & $<0.550$ & $<0.550$ \\
\hline 66 & $148.26+05.26$ & $04166+5719$ & & TW Cam & 4800 & 200 & 0.0 & 0.25 & GLG00 & 0.622 & 0.576 & 0.773 \\
\hline 67 & $174.77-12.19$ & $04440+2605$ & & RV Tau & 4500 & 200 & 0.0 & 0.25 & GLG00 & 0.599 & 0.557 & 0.639 \\
\hline 68 & $188.06+01.30$ & $06054+2237$ & 41870 & SS Gem & 5400 & 150 & 0.2 & 0.2 & GLG97b $^{9)}$ & 0.623 & 0.585 & 0.722 \\
\hline 69 & $195.41-03.42$ & $06034+1354$ & & DY Ori & 5900 & 200 & 1.5 & 0.2 & GLG97a9) $^{9}$ & $<0.550<$ & $<0.550$ & $<0.550$ \\
\hline 70 & $199.39-04.56$ & $06072+0953$ & & CT Ori & 5750 & 150 & 1.0 & 0.2 & GLG97b $^{9}$ & $<0.550<$ & $<0.550$ & 0.550 \\
\hline 71 & $217.80+09.95$ & $07331+0021$ & & AI CMi & 4500 & & 0.0 & & KP96 & 0.599 & & \\
\hline 72 & $226.14+04.15$ & 07284-0940 & 59693 & U Mon & 5000 & 200 & 0.0 & 0.25 & GLG00 & 0.645 & 0.599 & 0.873 \\
\hline 73 & $253.02-03.00$ & $08011-3627$ & & AR Pup & 6300 & 200 & 1.5 & 0.2 & GLG97a9) $^{9}$ & $<0.550<$ & $<0.550$ & $<0.550$ \\
\hline 74 & $282.42-09.24$ & $09256-6324$ & $82084 / 5$ & IW Car & 6700 & 200 & 2.0 & 0.25 & GRL94 & $<0.550<$ & $<0.550$ & $<0.550$ \\
\hline 75 & $293.17-17.24$ & $09538-7622$ & & & 5500 & 250 & 1.0 & 0.5 & MvWLE05 & $<0.550<$ & $<0.550$ & 0.554 \\
\hline 76 & $295.25+16.82$ & $12067-4508$ & 105578 & RU Cen & 6000 & 250 & 1.0 & 0.50 & MvWW02 & $<0.550<$ & $<0.550$ & 0.574 \\
\hline 77 & $297.87+13.36$ & $12185-4856$ & 107439 & SX Cen & 6250 & 250 & 1.5 & 0.50 & MvWW02 & $<0.550<$ & $<0.550$ & $<0.550$ \\
\hline 78 & $313.90-08.68$ & $14524-6838$ & 131356 & EN TrA & 6150 & 75 & 1.25 & 0.25 & vW972) & $<0.550<$ & $<0.550$ & $<0.550$ \\
\hline 79 & $330.84+57.77$ & $13467-0141$ & 120408 & CE Vir & 4300 & 100 & 0.3 & 0.2 & GLG97b $^{9)}$ & 0.550 & $<0.550$ & 0.555 \\
\hline 80 & $331.86-13.78$ & $17250-5951$ & & UY Ara & 5500 & 200 & 0.3 & 0.25 & GLG00 & 0.610 & 0.559 & 0.693 \\
\hline 81 & $339.79-04.68$ & $17038-4815$ & & & 4750 & 250 & 0.5 & 0.5 & MvWLE05 & $0.550<$ & $<0.550$ & 0.599 \\
\hline 82 & $344.12+26.45$ & & & BT Lib & 5800 & 200 & 1.4 & 0.25 & GLG00 ${ }^{10)}$ & $<0.550<$ & $<0.550$ & $<0.550$ \\
\hline 83 & $345.49-04.99$ & $17243-4348$ & & LR Sco & 6750 & 250 & 0.25 & 0.5 & MvWLE05 ${ }^{4)}$ & 0.939 & 0.604 & $>0.940$ \\
\hline 84 & $345.54+10.26$ & $16230-3410$ & & & 6250 & 250 & 1.0 & 0.5 & MvWLE05 & $0.550<$ & $<0.550$ & 0.599 \\
\hline 85 & $345.65-04.69$ & $17233-4330$ & & & 6250 & 250 & 1.5 & 0.5 & MvWLE05 & $<0.550<$ & $<0.550$ & $<0.550$ \\
\hline
\end{tabular}


Table 1. continued.

\begin{tabular}{|c|c|c|c|c|c|c|c|c|c|c|c|c|}
\hline $\begin{array}{l}\text { No. } \\
\text { (1) }\end{array}$ & $\begin{array}{ll}l & b \\
(2)\end{array}$ & $\begin{array}{l}\text { IRAS } \\
\text { (3) }\end{array}$ & $\begin{array}{l}\text { HD } \\
(4)\end{array}$ & $\begin{array}{l}\text { Other name } \\
\text { (5) }\end{array}$ & $\begin{array}{l}T_{\text {eff }} \\
\text { (6) }\end{array}$ & $\begin{array}{l}\text { Err. } 1 \\
(7)\end{array}$ & $\begin{array}{l}\log g \\
(8)\end{array}$ & $\begin{array}{l}g \text { Err. } \\
(9)\end{array}$ & $\begin{array}{l}\text { Notes } \\
(10)\end{array}$ & $\begin{array}{l}M_{\star} \\
(11)\end{array}$ & $\begin{array}{r}M_{\star \min } \\
(12)\end{array}$ & $\begin{array}{r}M_{\star \max } \\
\text { (13) }\end{array}$ \\
\hline \multicolumn{13}{|c|}{ suspected RV Tau stars from Maas et al. 2005} \\
\hline 86 & $033.59-07.22$ & $19157-0247$ & & LS IV -02 29 & 7750 & 250 & 1.0 & 0.5 & MvWLE05 & 0.578 & $<0.550$ & 0.894 \\
\hline 87 & $039.02-03.49$ & $19125+0343$ & & LS IV +0318 & 7750 & 250 & 1.0 & 0.5 & MvWLE05 & 0.578 & $<0.550$ & 0.894 \\
\hline 88 & $254.58+12.94$ & $09060-2807$ & & BZ Pyx & 6500 & 250 & 1.5 & 0.5 & MvWLE05 & $<0.550$ & $<0.550$ & 0.550 \\
\hline 89 & $265.50+00.39$ & $08544-4431$ & & & 7250 & 250 & 1.5 & 0.5 & MvWLE05 & $<0.550$ & $<0.550$ & 0.554 \\
\hline 90 & $271.51-00.50$ & 09144-4933 & & & 5750 & 250 & 0.5 & 0.5 & MvWLE05 & 0.574 & $<0.550$ & 0.860 \\
\hline 91 & $327.82+00.63$ & $15469-5311$ & & & 7500 & 250 & 1.5 & 0.5 & MvWLE05 & $<0.550$ & $<0.550$ & 0.557 \\
\hline \multicolumn{13}{|c|}{ R CrB stars } \\
\hline 92 & $002.41+07.51$ & & & V2552 Oph & 6750 & 250 & 0.5 & 0.5 & RL03 ${ }^{11)}$ & 0.660 & $0.554>$ & $>0.940$ \\
\hline 93 & $002.52-05.97$ & $18119-2943$ & 317333 & VZ Sgr & 7000 & 250 & 0.5 & 0.5 & AGLO0 & 0.722 & $0.557>$ & $>0.940$ \\
\hline 94 & $004.43-19.45$ & $19132-3336$ & 180093 & RY Sgr & 7250 & 250 & 0.75 & 50.5 & AGL00 & 0.616 & $0.551>$ & $>0.940$ \\
\hline 95 & $005.81-03.78$ & $18103-2547$ & & V3795 Sgr & 8000 & 250 & 1.0 & 0.5 & AGL00 & 0.599 & $<0.550$ & 0.933 \\
\hline 96 & $008.31-05.24$ & $18211-2417$ & & GU Sgr & 6250 & 250 & 0.5 & 0.5 & AGL00 & 0.614 & $0.551>$ & $>0.940$ \\
\hline 97 & $023.83-02.92$ & $18425-0928$ & & FH Sct & 6250 & 250 & 0.25 & 50.5 & AGL00 & 0.833 & $0.564>$ & $>0.940$ \\
\hline \multirow[t]{2}{*}{98} & $045.05+50.98$ & $15465+2818$ & 141527 & $\mathrm{R} \mathrm{CrB}$ & 6750 & 250 & 0.5 & 0.5 & AGL00 & 0.660 & $0.554>$ & $>0.940$ \\
\hline & & & & & 7000 & 150 & 0.5 & 0.25 & RGA90 & 0.722 & $0.611>$ & $>0.940$ \\
\hline 99 & $070.45+02.20$ & $19577+3351$ & & V482 Cyg & 6500 & 250 & 0.5 & 0.5 & AGLO0 & 0.629 & $0.552>$ & $>0.940$ \\
\hline 100 & $109.52-00.39$ & $23001+5920$ & & UV Cas & 7250 & 250 & 0.5 & 0.5 & AGLO0 & 0.841 & $0.563>$ & $>0.940$ \\
\hline 101 & $149.84+01.12$ & & 25878 & XX C & 7250 & 250 & 0.75 & 50.5 & AGL00 & 0.616 & $0.551>$ & $>0.940$ \\
\hline 102 & $188.86-04.42$ & $05461+1903$ & 247925 & SU Tau & 6500 & 250 & 0.5 & 0.5 & AGL00 & 0.629 & $0.552>$ & $>0.940$ \\
\hline 103 & $279.06+20.12$ & & & UX Ant & 7000 & 250 & 0.5 & 0.5 & AGL00 & 0.722 & $0.557>$ & $>0.940$ \\
\hline 104 & $301.74+08.32$ & $12404-5415$ & & UW Cen & 7500 & 250 & 1.0 & 0.5 & AGL00 & 0.563 & $<0.550$ & 0.841 \\
\hline 105 & $304.42-02.68$ & $13025-6514$ & & Y Mus & 7250 & 250 & 0.75 & 50.5 & AGL00 & 0.616 & $0.551>$ & $>0.940$ \\
\hline 106 & $307.96+08.29$ & $13224-5359$ & & DY Cen & 19500 & 500 & 2.15 & 50.1 & JH93 & 0.902 & 0.853 & 0.938 \\
\hline 107 & $327.22-06.92$ & $16200-5913$ & & RT Nor & 7000 & 250 & 1.5 & 0.5 & AGLO0 & $<0.550$ & $<0.550$ & 0.552 \\
\hline 108 & $332.44-03.57$ & $16287-5309$ & & RZ Nor & 6750 & 250 & 0.75 & 50.5 & AGL00 & 0.581 & $<0.550$ & 0.895 \\
\hline 109 & $347.53-14.14$ & $18151-4634$ & & RS Tel & 6750 & 250 & 1.25 & 50.5 & AGL00 & $<0.550$ & $<0.550$ & 0.564 \\
\hline 110 & $357.66-15.65$ & $18441-3812$ & 173539 & $\mathrm{~V} \mathrm{CrA}$ & 6250 & 250 & 0.5 & 0.5 & AGL00 & 0.614 & $0.551>$ & $>0.940$ \\
\hline \multicolumn{13}{|c|}{ extreme helium stars } \\
\hline 111 & $006.01+26.02$ & & & V2205 Oph & 22700 & 1200 & 2.55 & 50.1 & JH92 & 0.715 & 0.705 & 0.721 \\
\hline 112 & $007.82+05.07$ & & & LS 4357 & 16130 & 500 & 2.00 & 00.25 & JHHJ98 & 0.675 & 0.605 & 0.913 \\
\hline \multirow[t]{2}{*}{113} & $020.91-08.31$ & & & V4732 Sgr & 9500 & 250 & 0.9 & 0.2 & PRL01 & 0.902 & $0.699>$ & $>0.940$ \\
\hline & & & & & 9000 & 250 & 1.0 & 0.5 & AGLO0 & 0.659 & $0.554>$ & $>0.940$ \\
\hline 114 & $024.41+12.50$ & & & V2244 Oph & 12750 & 250 & 1.75 & 50.25 & PRL01 & 0.612 & 0.558 & 0.732 \\
\hline 115 & $026.55+10.09$ & & & No Ser & 11750 & 250 & 2.30 & 00.4 & PRL01 & $<0.550$ & $<0.550$ & 0.553 \\
\hline 116 & $031.33+33.28$ & & & V652 Her & 24550 & 500 & 3.68 & 80.05 & JHH99 & $<0.550$ & $<0.550<$ & $<0.550$ \\
\hline \multirow[t]{2}{*}{117} & $049.87-25.21$ & & & FQ Aqr & 8750 & 250 & 0.75 & 50.25 & PRL01 & 0.908 & $0.659>$ & $>0.940$ \\
\hline & & & & & 8500 & 250 & 1.5 & 0.5 & AGLOO & 0.550 & $<0.550$ & 0.611 \\
\hline 118 & $068.90+04.76$ & & 225642 & V1920 Cyg & 16180 & 500 & 2.0 & 0.25 & PLRJ04 & 0.681 & 0.606 & 0.917 \\
\hline 119 & $222.95-04.18$ & & & LS 99 & 15330 & 500 & 1.90 & 00.25 & JHHJ98 & 0.688 & 0.607 & 0.919 \\
\hline 120 & $235.21+54.44$ & & & DN Leo & 16800 & 600 & 2.55 & 50.2 & Heb83 & 0.553 & 0.550 & 0.560 \\
\hline 121 & $309.95-04.25$ & & & BX Cir & 23300 & 700 & 3.35 & 50.10 & DJH98 & $<0.550$ & $<0.550<$ & $<0.550$ \\
\hline 122 & $317.65+14.18$ & & 124448 & V821 Cen & 15500 & 800 & 2.1 & 0.2 & PLRJ04 & 0.610 & 0.576 & 0.634 \\
\hline 123 & $338.13-18.71$ & & 168476 & PV Tel & 14000 & 500 & 1.5 & 0.2 & WS81 & $>0.940$ & 0.845 & $>0.940$ \\
\hline 124 & $344.19-08.84$ & & & CD-46 11775 & 18000 & 700 & 2.00 & 00.1 & Jef93 & 0.910 & 0.883 & 0.930 \\
\hline 125 & $348.17+17.78$ & & 144941 & & 23200 & 500 & 3.90 & 00.2 & HJ97 & $<0.550$ & $<0.550<$ & $<0.550$ \\
\hline
\end{tabular}

Notes in Col. 8:

${ }^{1}$ Average from Table 4 of Luck et al. (1990); ${ }^{2}$ average from two models of Van Winckel (1997); ${ }^{3}$ an error of $500 \mathrm{~K}$ for $T_{\text {eff }}$ has been assumed;

${ }^{4}$ average of models for different time of observations; ${ }^{5}$ average of models for two spectra; ${ }^{6}$ average from two phases; ${ }^{7}$ average from two models of Reyniers et al. (2004b); ${ }^{8}$ average from two models of Van Winckel et al. (1996a); ${ }^{9}$ avearage value; ${ }^{10} \log (g)$ is averaged from two epoches; ${ }^{11}$ errors assumed are the same as in Asplund et al. (2000).

of atmospheric parameters are not independent. The paper by AFGM01 was suggested by the work of PGS00. It is not quite clear how the atmospheric parameters were determined. The analysis of RDM03 is based on non-LTE, fully blanketed atmospheric models and the same spectroscopic material as used in the LTE analysis by MRK02. Hence preference is given to 
Table 1. continued. The abbreviations used in Col. (10).

AFGM01: Arellano Ferro et al. (2001)
AGL00: Asplund et al. (2000)
CDK94: Conlon et al. (1994)
CDK91: Conlon et al. (1991)
CTM93: Conlon et al. (1993)
DJH98: Drilling et al. (1998)
DvWW98: Decin et al. (1998)
GP03: Gauba \& Parthasarathy (2003)
GAFP97: Giridhar et al. (1997)
GLG98: Giridhar et al. (1998)
GLG00: Giridhar et al. (2000)
GRL94: Giridhar et al. (1994)
GLG97a: Gonzalez et al. (1997a)
GLG97b: Gonzalez et al. (1997b)
GW92: Gonzalez \& Wallerstein (1992)
HDK96: Hambly et al. (1996)
HJ97: Harrison \& Jeffery (1997)
H83: Heber (1983)
HR03: Hrivnak \& Reddy (2003)
J93: Jeffery (1993)
JH92: Jeffery \& Heber (1992)
JH93: Jeffery \& Heber (1993)
JHHJ98: Jeffery et al. (1998)
JHH99: Jeffery et al. (1999)

KDK97: Kendall et al. (1997)

KL86: Kilkenny \& Lydon (1986)

K95: Klochkova (1995)

KP96: Klochkova \& Panchuk (1996)

KPT02: Klochkova et al. (2002b)

KSP00a: Klochkova et al. (2000a)

KSP00b: Klochkova et al. (2000b)

KSP99: Klochkova et al. (1999)

KYM02: Klochkova et al. (2002a)

K73: Kodaira (1973)

LB84: Luck \& Bond (1984)

LBL90: Luck et al. (1990)

LLB83: Luck et al. (1983)

MvWW02: Maas et al. (2002)

MvWLE05: Maas et al. (2005)

MCD92: McCausland et al. (1992)

MH98: Moehler \& Heber (1998)

MRK02: Mooney et al. (2002)

MDS04: Munn et al. (2004)

NHK94: Napiwotzki et al. (1994)

PGS00: Parthasarathy et al. (2000)

PLM04: Pereira et al. (2004)

PRL01: Pandey et al. (2001)

PLRJ04: Pandey et al. (2004)
RGA90: Rao et al. (1990)

RGL02: Rao et al. (2002)

RL03: Rao \& Lambert (2003)

RBH99: Reddy et al. (1999)

RH99: Reddy \& Hrivnak (1999)

RLG02: Reddy et al. (2002)

RPS96: Reddy et al. (1999)

RDvW04: Reyniers et al. (2004a)

RvW01: Reyniers \& Van Winckel (2001)

RvWG04: Reyniers et al. (2004b)

RDM03: Ryans et al. (2003)

SPG99: Sivarani et al. (1999)

TPJ00: Thévenin et al. (2000)

vW95: Van Winckel (1995)

vW97: Van Winckel (1997)

vWOT96: van Winckel et al. (1996a)

vWR00: Van Winckel \& Reyniers (2000)

vWWW96: Van Winckel et al. (1996b)

VSL98: Veen et al. (1998)

WvWB91: Waelkens et al. (1991)

WvWTW92: Waelkens et al. (1992)

WS81: Walker \& Schönberner (1981)

ZKP95: Zaćs et al. (1995)

ZKP96: Zaćs et al. (1996) work by RDM03. Their values consistently point to a higher mass of the star.

Object 19: IRAS 19475+3119. Since the paper of KPT02 is based on spectra with relatively low resolution (15000), the analysis by AFGM01 is probably more reliable.

Object 20: IRAS 17436+5003. Both analysis by KPT02 and LBL90 result in a massive post-AGB star. KPT02 spectra have lower resolution than those of LBL90, so we adopted the data from the latter.

Object 22: IRAS 22223+4327. Both papers by vWR00 and DvWW98 are based on the same spectroscopical material and the same methods of analysis. We adopted the data from the more recent paper $\mathrm{vWR} 00$.

Object 24: IRAS 22272 $+\mathbf{5 4 3 5}$. Both papers by ZKP95 and RLG02 give consistent atmospheric parameters but differ in derived abundances, particularly of $\mathrm{Fe}$. Other determinations are based on relatively few lines $(\mathrm{C}, \mathrm{N})$ or on just one line $(\mathrm{Zn}, \mathrm{O})$ and may be in error. The paper by RLG02 is based on higher quality material so we used their results.

Object 25: IRAS 23304+6147 The vWR00 analysis is based on much higher resolution spectra $(60000)$ compared to that of KSP00 (15000), so we used the results from vWR00.

Object 29: IRAS 04296 +3429 . The works by vWR00 and DvWW98 are based on the same spectroscopical material and the same methods of analysis. Data from vWR00 were adopted. KSP99 spectra have lower resolution.
Object 35: IRAS 07134+1005. All analyses of HD 56126 give similar values of atmospheric parameters. The papers by HR03 and vWR00 are based on a higher quality spectrum than the paper by K95, yet there is a difference in the absolute determination of the carbon abundance. The remaining abundances are similar. Data from vWR00 were adopted.

Object 37: IRAS 06530-0213. The analysis of RvWG04 is based on higher quality spectroscopic material than HR03, so their results were used.

Object 50: IRAS 12538-2611. The analysis of GAFP97 is based on higher quality spectra and more lines are used for the determination of the chemical abundances, so their values were preferred.

Object 52: BPS CS 22877-0023. Both analyses lead to uncertain determinations of atmospheric parameters, but MH98 used a larger number of methods, so we adopted their values.

Object 54: LS 3591. We adopted the VSL98 results since only their work gives chemical abundances.

Object 57: V453 Oph and object 60: HD 216457. The abundance analysis of RDvW04 is based on higher quality spectra and a wider spectral range than that of GLG00 and GLG98, so we adopted the results from RDvW04.

Object 98: IRAS 15465+2818. The paper by AGL00 is the only one that gives chemical abudances.

Object 113: V4732 Sgr and object 117: FQ Aqr. The paper of PRL01 gives a consistent set of abundances for the 
Table 2. Chemical composition of our of post-AGB sample.

\begin{tabular}{|c|c|c|c|c|c|c|c|}
\hline $\begin{array}{l}\text { No } \\
\text { (1) }\end{array}$ & $\begin{array}{l}\text { Name } \\
\text { (2) }\end{array}$ & $\begin{array}{r}\epsilon(\mathrm{C}) \\
(3)\end{array}$ & $\begin{array}{r}\epsilon(\mathrm{N}) \\
\quad(4)\end{array}$ & $\begin{array}{r}\epsilon(\mathrm{O}) \\
\quad(5)\end{array}$ & $\begin{array}{r}\epsilon(\mathrm{S}) \\
(6)\end{array}$ & $\begin{array}{r}\epsilon(\mathrm{Fe}) \\
\quad(7)\end{array}$ & $\begin{array}{r}\epsilon(\mathrm{Zn}) \\
(8)\end{array}$ \\
\hline \multirow[t]{2}{*}{1} & $006.72-10.37$ & 7.93 & 7.34 & 8.29 & 7.17 & 6.89 & 4.37 \\
\hline & & 8.01 & 7.61 & 8.47 & 7.06 & 6.94 & 4.17 \\
\hline 2 & $007.96+26.71$ & 8.35 & 8.55 & 8.85 & 7.30 & 7.10 & 4.76 \\
\hline \multirow[t]{2}{*}{3} & $013.23+12.17$ & 8.58 & 7.65 & 8.41 & 7.22 & 7.12 & 4.52 \\
\hline & & 8.30 & & 8.33 & 7.29 & 6.92 & \\
\hline 4 & $016.45-50.43$ & $<6.80$ & 7.00 & 8.00 & & & \\
\hline \multirow[t]{2}{*}{5} & $023.98-21.04$ & 8.98 & 8.37 & 8.96 & 6.86 & 6.91 & \\
\hline & & 9.08 & 8.32 & 9.13 & 7.36 & 7.17 & \\
\hline \multirow[t]{2}{*}{6} & $029.18-21.26$ & 7.50 & 7.65 & 8.51 & 7.40 & & \\
\hline & & 6.70 & 7.80 & 8.80 & 6.60 & 6.70 & \\
\hline 7 & $030.60-21.53$ & 7.03 & 7.11 & 7.51 & 6.32 & 6.38 & 3.80 \\
\hline 8 & $031.33-43.48$ & 6.40 & 7.50 & 8.20 & 6.70 & 6.20 & \\
\hline 9 & $033.16-48.12$ & $<6.10$ & 6.80 & 7.90 & $<6.50$ & & \\
\hline \multirow[t]{3}{*}{10} & $035.62-04.96$ & 8.74 & 9.10 & 9.37 & 7.38 & 7.35 & \\
\hline & & 9.17 & & & & 7.37 & \\
\hline & & $<7.52$ & & & & $<7.00$ & $<4.35$ \\
\hline 11 & $040.51-10.09$ & 7.74 & & & & 6.40 & 4.07 \\
\hline \multirow[t]{2}{*}{12} & $043.06+32.36$ & 6.80 & $<6.70$ & & & & \\
\hline & & 7.01 & 7.02 & 7.90 & & & \\
\hline 13 & $043.23-57.13$ & 9.02 & 8.69 & 8.76 & 7.57 & 6.78 & \\
\hline \multirow[t]{4}{*}{14} & $050.67+19.79$ & 7.19 & 7.43 & 8.47 & & & \\
\hline & & 7.12 & 7.47 & 8.36 & & & \\
\hline & & 6.92 & 7.22 & 8.23 & 6.33 & 6.90 & \\
\hline & & 7.04 & 7.52 & 8.35 & 7.25 & & \\
\hline 15 & $051.43+23.19$ & 8.30 & 8.29 & 8.66 & 7.01 & 7.08 & 4.27 \\
\hline 16 & $052.73+50.79$ & 7.40 & 7.30 & 8.10 & & 5.50 & \\
\hline 17 & $053.84+20.18$ & 8.27 & 7.66 & 8.74 & 6.96 & 6.72 & 4.60 \\
\hline 18 & $066.18+18.58$ & 7.25 & & 9.20 & 6.88 & 6.89 & \\
\hline \multirow[t]{2}{*}{19} & $067.16+02.73$ & 8.46 & 9.02 & 9.29 & 7.37 & 7.27 & 4.41 \\
\hline & & 8.22 & & 8.93 & 7.50 & 7.27 & \\
\hline \multirow[t]{2}{*}{20} & $077.13+30.87$ & 8.45 & 8.92 & 9.01 & 7.68 & 7.17 & 4.89 \\
\hline & & 8.52 & 8.39 & 9.15 & 7.46 & 7.26 & 4.63 \\
\hline 21 & $080.17-06.50$ & 8.69 & 9.38 & 8.68 & 7.09 & 6.92 & 3.91 \\
\hline \multirow[t]{2}{*}{22} & $096.75-11.56$ & 8.58 & 7.84 & 8.50 & 6.95 & 7.20 & \\
\hline & & 8.63 & 7.88 & 8.50 & 6.85 & 7.07 & \\
\hline 23 & $098.41-16.73$ & 8.20 & 8.30 & 8.80 & 7.30 & 4.55 & \\
\hline \multirow[t]{2}{*}{24} & $103.35-02.52$ & 8.69 & 7.68 & 8.48 & 6.89 & 6.69 & 4.16 \\
\hline & & 7.37 & & 8.77 & & 7.02 & 6.30 \\
\hline \multirow[t]{2}{*}{25} & $113.86+00.59$ & 8.70 & 7.68 & 8.24 & 6.98 & 6.72 & \\
\hline & & 8.89 & 8.69 & 9.03 & 7.05 & 6.86 & 4.68 \\
\hline 26 & $123.57+16.59$ & 8.32 & 7.70 & 8.24 & & 7.19 & \\
\hline 27 & $133.73+01.50$ & 8.84 & 8.67 & & 7.07 & 7.03 & \\
\hline 28 & $161.98+19.59$ & 8.19 & & 8.47 & & 5.95 & 3.24 \\
\hline \multirow[t]{3}{*}{29} & $166.24-09.05$ & 8.71 & 7.76 & & 7.02 & 6.89 & \\
\hline & & 8.55 & 7.96 & 8.22 & 6.80 & 6.66 & 3.84 \\
\hline & & 8.81 & 7.84 & & 7.13 & 6.82 & \\
\hline 30 & $172.95-05.50$ & $<6.70$ & 7.30 & 7.60 & $<6.29$ & $<6.70$ & \\
\hline 31 & $173.86-82.41$ & $<6.90$ & 7.40 & 8.20 & $<6.50$ & $<6.20$ & \\
\hline 32 & $188.86-14.29$ & 8.81 & 8.24 & 8.43 & 6.87 & 6.75 & 3.74 \\
\hline 33 & $196.19-12.14$ & 8.73 & 7.83 & 8.57 & 6.64 & 6.66 & \\
\hline 34 & $204.67+07.57$ & 8.14 & 7.55 & 8.47 & 6.19 & 3.05 & \\
\hline \multirow[t]{3}{*}{35} & $206.75+09.99$ & 8.09 & 7.87 & 8.40 & 6.49 & 6.50 & \\
\hline & & 8.65 & 7.84 & 8.67 & 6.61 & 6.51 & 3.97 \\
\hline & & 8.63 & 8.00 & 8.49 & 6.84 & 6.50 & \\
\hline 36 & $208.93+17.07$ & 8.76 & 7.97 & & 6.98 & 7.06 & \\
\hline \multirow[t]{2}{*}{37} & $215.44-00.13$ & 8.72 & 7.97 & 8.30 & & 6.59 & \\
\hline & & 9.09 & & 8.64 & 6.97 & 7.03 & \\
\hline
\end{tabular}

Table 2. continued.

\begin{tabular}{|c|c|c|c|c|c|c|c|}
\hline $\begin{array}{l}\text { No } \\
\text { (1) }\end{array}$ & $\begin{array}{l}\text { Name } \\
\text { (2) }\end{array}$ & $\begin{array}{r}\epsilon(\mathrm{C}) \\
\quad(3)\end{array}$ & $\begin{array}{r}\epsilon(\mathrm{N}) \\
(4)\end{array}$ & $\begin{array}{r}\epsilon(\mathrm{O}) \\
\quad(5)\end{array}$ & $\begin{array}{r}\epsilon(\mathrm{S}) \\
(6)\end{array}$ & $\begin{array}{r}\epsilon(\mathrm{Fe}) \\
\quad(7)\end{array}$ & $\begin{array}{r}\epsilon(\mathrm{Zn}) \\
\quad(8)\end{array}$ \\
\hline 38 & $218.97-11.76$ & 8.62 & 7.82 & 8.72 & 7.04 & 4.00 & \\
\hline 39 & $236.57-05.37$ & 7.94 & 7.56 & & 6.39 & 6.67 & 3.60 \\
\hline 40 & $260.83-0$ & 8.64 & 7.76 & 8.52 & 7.07 & 7.12 & 4.54 \\
\hline 41 & $264.55+72.47$ & 8.46 & 7.79 & 8.37 & & 6.30 & \\
\hline 42 & $266.85+22.93$ & 8.41 & 8.07 & 8.64 & 6.82 & 2.86 & \\
\hline 43 & $269.97-3$ & 8.25 & 8.24 & 8.82 & 6.90 & 5.82 & \\
\hline 44 & $290.54-0$ & 8.51 & 7.98 & 8.52 & 7.09 & 7.61 & 4.29 \\
\hline 45 & 293.0 & 9.55 & 8.50 & 8.97 & & 7.50 & \\
\hline 46 & & 7.11 & 7.31 & 7.81 & 6.14 & 6.30 & \\
\hline 47 & 298. & 8.54 & 8.16 & 8.80 & 6.97 & 7.43 & 4.31 \\
\hline 48 & $298.30+08.6$ & 8.11 & 7.76 & 8.70 & 7.15 & 6.77 & 3.78 \\
\hline 49 & 299.0 & 6.70 & 7.60 & 7.60 & 5.90 & $<6.70$ & \\
\hline \multirow[t]{2}{*}{50} & $304.34+36.40$ & 7.28 & & 8.59 & 6.53 & 6.42 & 3.76 \\
\hline & & 7.52 & 7.34 & 8.57 & 6.15 & 6.31 & \\
\hline 51 & $309.07+15.18$ & 7.46 & 7.30 & 8.17 & 6.53 & 5.70 & 3.44 \\
\hline \multirow[t]{2}{*}{52} & $317.11+53.11$ & $<6.80$ & 6.80 & $<7.90$ & & & \\
\hline & & $<6.16$ & 6.93 & 7.98 & & & \\
\hline 53 & $325.04+08.65$ & 7.94 & 7.78 & 8.52 & 6.83 & 6.81 & \\
\hline \multirow[t]{2}{*}{54} & $326.77-07.49$ & & & & & & \\
\hline & & & 8.00 & 9.30 & & 6.70 & \\
\hline 55 & 330. & & 7.20 & 8.30 & & 5.50 & \\
\hline 56 & 345.5 & 7.83 & 7.57 & 8.60 & & 7.56 & \\
\hline \multicolumn{8}{|c|}{ RV Tau stars } \\
\hline \multirow[t]{2}{*}{57} & $020.72+17.57$ & 6.61 & & 6.93 & & 5.34 & \\
\hline & & 6.08 & & 7.62 & & 5.29 & 2.50 \\
\hline 58 & $021.48+28.63$ & 8.07 & & 8.33 & 7.25 & 6.65 & 3.91 \\
\hline 59 & 026 & 8.24 & & 63 & 7.21 & 5.38 & 4.50 \\
\hline \multirow[t]{3}{*}{60} & $044.10-61.56$ & 7.26 & & 8.53 & 6.42 & 6.36 & 3.55 \\
\hline & & & & 8.54 & 6.16 & 6.50 & \\
\hline & & 6.80 & & 7.88 & & 5.90 & 3.11 \\
\hline 61 & & 8.50 & & 8.65 & 6.90 & 6.10 & 3.69 \\
\hline 62 & & 8.15 & & 29 & 7.58 & 7.01 & 4.41 \\
\hline 63 & 058. & 8.85 & 8.83 & 9.15 & 7.47 & 7.24 & 4.46 \\
\hline 64 & $060.73+06.94$ & 8.19 & 7.82 & 8.83 & 6.60 & .71 & 3.90 \\
\hline 65 & $076.75-11.78$ & $<6.15$ & & 34 & 6.36 & 6.10 & 3.26 \\
\hline 66 & 148 & 8.79 & & 8.44 & 7.19 & 7.00 & 4.28 \\
\hline 67 & 174.7 & 9.23 & & 8.69 & & 7.07 & 4.64 \\
\hline 68 & & 8.15 & 7.39 & 8.38 & 6.96 & 63 & 4.59 \\
\hline 69 & 195.4 & 8.38 & & 8.94 & 7.37 & 5.20 & 4.81 \\
\hline 70 & 199.3 & 8.02 & & 8.29 & 6.85 & 5.63 & 3.99 \\
\hline 71 & $217.80+09.95$ & & & & & 6.32 & 4.17 \\
\hline 72 & $226.14+04.15$ & 8.37 & & 8.46 & 7.09 & 6.71 & 3.91 \\
\hline 73 & 253.0 & & & 9.01 & 7.65 & 64 & \\
\hline 74 & 282 & 8.87 & & & 7.59 & .45 & 4.60 \\
\hline 75 & $7-17.24$ & 8.32 & & 9.13 & 7.03 & 6.90 & 4.10 \\
\hline 76 & $295.25+16.82$ & 8.14 & 7.72 & 8.57 & 6.53 & 5.63 & 3.60 \\
\hline 77 & 297.8 & 8.50 & 8.42 & 9.00 & 7.13 & 6.37 & 4.06 \\
\hline 78 & 313.9 & 8.21 & 7.83 & 8.29 & 6.65 & 7.00 & 4.14 \\
\hline 79 & $330.84+57.77$ & & & & & 6.31 & 3.87 \\
\hline 80 & $331.86-13.78$ & 8.39 & & & 7.25 & 6.48 & 4.33 \\
\hline 81 & $339.79-04.68$ & 8.82 & & & & 6.00 & 3.40 \\
\hline 82 & $344.12+26.45$ & 7.30 & & 8.53 & 6.48 & 6.32 & 3.56 \\
\hline 83 & & 8.32 & 7.72 & 8.72 & 7.33 & 7.50 & 4.80 \\
\hline 84 & $345.54+10.26$ & 8.02 & 7.42 & 8.43 & 7.03 & 6.80 & 4.20 \\
\hline 85 & $345.65-04.69$ & 8.32 & 8.12 & 8.53 & 7.43 & 6.50 & 4.30 \\
\hline
\end{tabular}


Table 2. continued.

\begin{tabular}{lllllll}
\hline \hline No name & $\epsilon(\mathrm{C})$ & $\epsilon(\mathrm{N})$ & $\epsilon(\mathrm{O})$ & $\epsilon(\mathrm{S})$ & $\epsilon(\mathrm{Fe})$ & $\epsilon(\mathrm{Zn})$ \\
$(1)(2)$ & $(3)$ & $(4)$ & $(5)$ & $(6)$ & $(7)$ & $(8)$ \\
\hline
\end{tabular}

\section{suspected RV Tau stars from Maas et al. 2005}

$\begin{array}{llllllll}86 & 33.59-07.22 & 8.42 & & 9.02 & & 7.60 & \\ 87 & 39.02-03.49 & 8.72 & & 9.03 & 7.53 & 7.20 & 4.70 \\ 88 & 254.58+12.94 & 7.62 & & & 6.53 & 6.80 & 4.00 \\ 89 & 265.50+00.39 & 8.22 & 7.82 & 8.33 & 7.53 & 7.20 & 4.70 \\ 90 & 271.51-00.50 & 8.22 & 8.12 & 8.53 & & 7.20 & \\ 91 & 327.82+00.63 & 8.82 & 8.52 & 8.83 & 7.93 & 7.50 & 4.90\end{array}$

\section{R CrB stars}

$\begin{array}{llllllll}92 & 002.41+07.51 & 9.11 & 8.42 & 8.60 & 6.70 & 6.40 & 4.16 \\ 93 & 002.52-05.97 & 8.80 & 7.60 & 8.70 & 6.70 & 5.80 & 3.90 \\ 94 & 004.43-19.45 & 8.90 & 8.50 & 7.90 & 7.30 & 6.70 & 4.50 \\ 95 & 005.81-03.78 & 8.80 & 8.00 & 7.50 & 7.40 & 5.60 & 4.10 \\ 96 & 008.31-05.24 & 8.80 & 8.70 & 8.20 & 7.00 & 6.30 & 4.40 \\ 97 & 023.83-02.92 & 8.80 & 8.79 & 7.70 & 7.00 & 6.30 & 4.10 \\ 98 & 045.05+50.98 & 9.20 & 8.40 & 9.00 & 6.80 & 6.50 & \\ & & & & & & & \\ 99 & 070.45+02.20 & 8.90 & 8.80 & 8.10 & 6.90 & 6.70 & 4.40 \\ 100 & 109.52-00.39 & 9.20 & 8.50 & 7.50 & 7.00 & 6.90 & 4.80 \\ 101 & 149.84+01.12 & 9.00 & 8.90 & 8.40 & 6.80 & 6.80 & \\ 102 & 188.86-04.42 & 8.80 & 8.50 & 8.40 & 6.50 & 6.10 & 3.60 \\ 103 & 279.06+20.12 & 8.90 & 8.30 & 8.80 & 6.20 & 6.20 & \\ 104 & 301.74+08.32 & 8.60 & 8.30 & 7.70 & 6.70 & 6.30 & 4.30 \\ 105 & 304.42-02.68 & 8.90 & 8.80 & 7.70 & 6.90 & 6.50 & 4.40 \\ 106 & 307.96+08.29 & 9.51 & 8.01 & 8.85 & 7.11 & 5.04 & \\ 107 & 327.22-06.92 & 8.90 & 9.10 & 8.40 & 7.70 & 6.80 & 4.70 \\ 108 & 332.44-03.57 & 8.90 & 8.70 & 8.90 & 6.80 & 6.60 & 4.40 \\ 109 & 347.53-14.14 & 8.90 & 8.80 & 8.30 & 6.80 & 6.40 & 4.30 \\ 110357.66-15.65 & 8.60 & 8.60 & 8.70 & 7.50 & 5.50 & 2.90\end{array}$

\section{extreme helium stars}

\begin{tabular}{lllllll}
$111006.01+26.02$ & 9.17 & 7.97 & 7.90 & 7.83 & 6.57 & \\
$112007.82+05.07$ & 9.38 & 8.16 & 9.39 & 7.12 & 6.84 & \\
$113020.91-08.31$ & 9.45 & 8.60 & 8.50 & 7.65 & 6.90 & \\
& 8.90 & 8.60 & & 7.10 & 6.30 & \\
$114024.41+12.50$ & 9.30 & 8.25 & 8.85 & 6.70 & 6.30 & \\
$115026.55+10.09$ & 9.00 & 8.50 & 8.40 & 6.90 & 6.70 & \\
$116031.33+33.28$ & & 8.93 & 7.54 & 7.44 & 7.40 & \\
$117049.87-25.21$ & 9.00 & 7.15 & 8.90 & 6.00 & 5.40 & \\
& 8.60 & 7.20 & 8.40 & 5.80 & 5.50 & 3.60 \\
$118068.90+04.76$ & 9.65 & 8.60 & 9.60 & 7.20 & 6.90 & 4.60 \\
$119222.95-04.18$ & 9.13 & 7.61 & 8.59 & 6.92 & 6.89 & \\
$120235.21+54.44$ & 9.54 & 8.11 & 8.10 & 7.12 & 6.49 & \\
$121309.95-04.25$ & 9.02 & 8.26 & 8.05 & 6.67 & 6.52 & \\
$122317.65+14.18$ & 9.40 & & & & 7.10 & 4.20 \\
$123338.13-18.71$ & 9.50 & 8.90 & 8.40 & 7.00 & 7.50 & \\
$124344.19-08.84$ & 9.54 & 8.33 & 9.06 & 7.13 & 6.78 & \\
$125348.17+17.78$ & 6.80 & 6.46 & 6.95 & & 6.38 & \\
\hline
\end{tabular}

sample of extreme helium stars analyzed here, so their results are preferred.

\section{Empirical diagrams}

\subsection{Choice of a metallicity indicator}

Since it is expected that yields are strongly dependent on "metallicity" or, better said, on the initial chemical composition of the star, we first have to choose a reasonable metallicity indicator. As stressed, e.g., by Mathis \& Lamers (1992) or Lambert (2004), the usual metallicity indicator in stellar atmospheres, Fe, cannot be used for post-AGB stars because of possible strong depletion in dust grains in a former stage and subsequent ejection of the grains (dust-gas separation). Oxygen, the most abundant "metal" and thus the best theoretical metallicity indicator, is possibly affected by nucleosynthesis on the AGB (ON cycle, hot bottom burning). From the list of elements for which we compiled the abundances, good metallicity indicators would be $\mathrm{S}$ and $\mathrm{Zn}$. The first one is an $\alpha$-element, like $\mathrm{O}$, and its abundance in the Galaxy is proportional to that of $\mathrm{O}$. For the second one, the nucleosynthesis mechanism is unknown a priori, but $\mathrm{Zn}$ appears to roughly follow $\mathrm{Fe}$ (Mishenina et al. 2002). Figure 1a shows $\epsilon(\mathrm{S})$ vs. $\epsilon(\mathrm{Zn})$ in our objects. The correlation is quite good (there is one outlier: V CrA, which also appears to be as an extreme object in many of the abundance-ratio diagrams of Asplund et al. 2000). The dispersion gives an idea of a realistic average abundance uncertainty: about 0.3 dex. Note that the data set spans a metallicity range of 2 dex. Figure $1 \mathrm{~b}$ shows that $\mathrm{S} / \mathrm{Zn}$ has a tendency to increase as $\epsilon(\mathrm{Zn})$ decreases (on average by 0.5 dex per dex). This is the well-known $\alpha$-enhancement observed in Population II stars (Norris et al. 2001). The effect of $\alpha$-enhancement on stellar parameters and stellar evolution is complex (Kim et al. 2002) and has not yet been investigated in AGB stars. We chose $\mathrm{S}$ as our principal metallicity indicator, since there are more stars with determinations of $\mathrm{S}$ abundances (113) than of $\mathrm{Zn}$ abundances (76). We keep $\mathrm{Zn}$ as a secondary indicator that might test the effect of $\alpha$-enhanced mixtures in the stars. We note, however, that the $\mathrm{Zn}$ abundance measurements often rely only on one line, which makes the evaluation of statistical errors difficult.

Figure 2 shows $\epsilon(\mathrm{O})$ vs. $\epsilon(\mathrm{S})$. The dispersion is such that obviously oxygen cannot be chosen as a metallicity indicator in our sample. The reason for this dispersion is not clear a priori. It can be due to nucleosynthesis and mixing affecting the oxygen abundance. But it could also be due to larger uncertainties in the oxygen abundance than was thought. Note that the R Cr B stars and extreme helium stars show the largest dispersion and the larger proportion of objects with $\mathrm{O} / \mathrm{S}$ smaller than solar.

\subsection{Nitrogen enhancement}

Figure 3 shows $\log \mathrm{N} / \mathrm{O}$ vs. $\epsilon(\mathrm{O})$. The objects from the $\mathrm{R} \mathrm{CrB}$ class and the extreme helium stars (which are represented by black circles) show different behaviour from the rest: they draw a clear anticorrelation between $\mathrm{N} / \mathrm{O}$ and $\epsilon(\mathrm{O})$. This diagram has also been constructed for planetary nebulae 

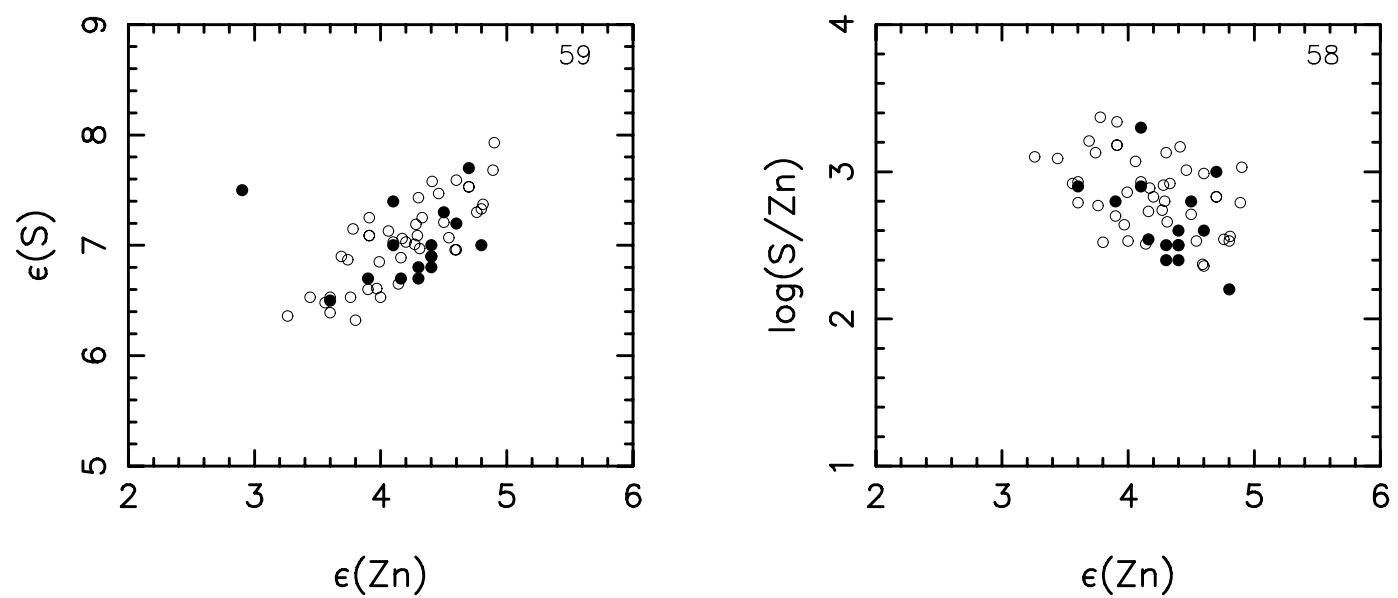

Fig. 1. a) (Left) The $\epsilon(\mathrm{S})$ vs. $\epsilon(\mathrm{Zn})$ relation in our post-AGB sample. b) (Right) The $\mathrm{S} / \mathrm{Zn}$ vs. $\epsilon(\mathrm{Zn})$ relation. Objects from the R CrB class and extreme helium stars are represented by black circles. The number of objects in our sample with available data is indicated in the top right corner of each panel.

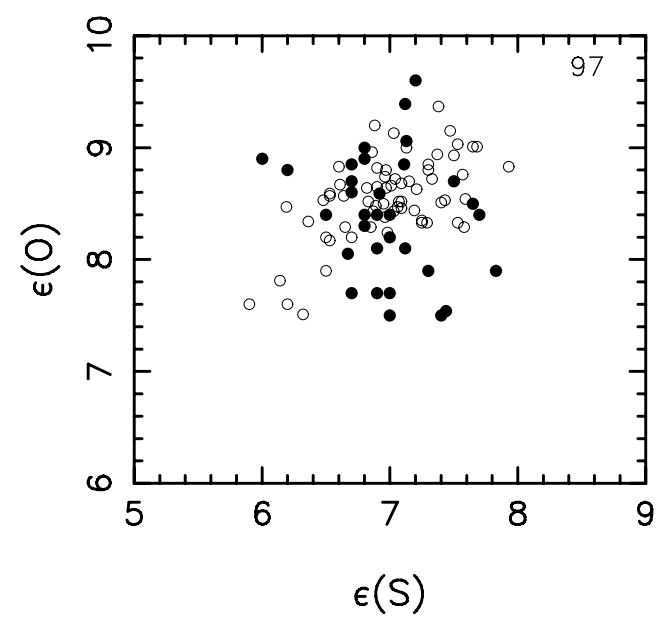

Fig. 2. The $\epsilon(\mathrm{O})$ vs. $\epsilon(\mathrm{S})$ relation.

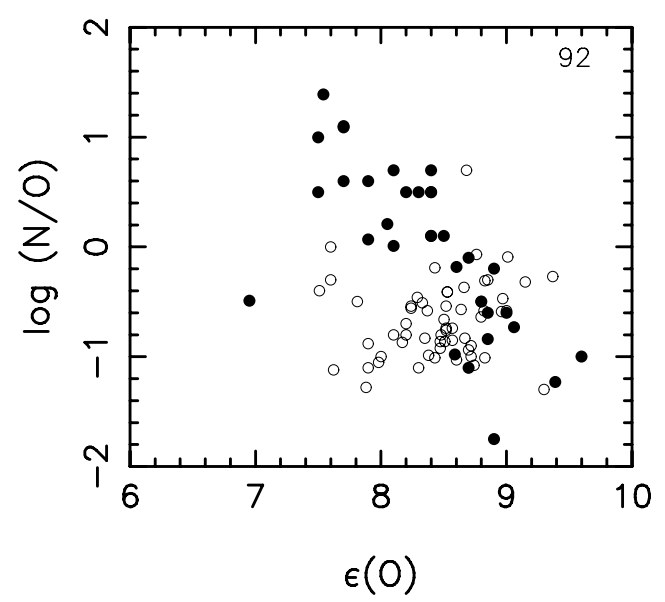

Fig. 3. The $\log \mathrm{N} / \mathrm{O}$ vs. $\epsilon(\mathrm{O})$ relation.

(e.g. Henry et al. 1989; Kingsburgh \& Barlow 1994; Leisy \& Dennefeld 1996) with different results depending on the authors and on the samples. Some claim not to see any anticorrelation. To our knowledge, never has the anticorrelation been

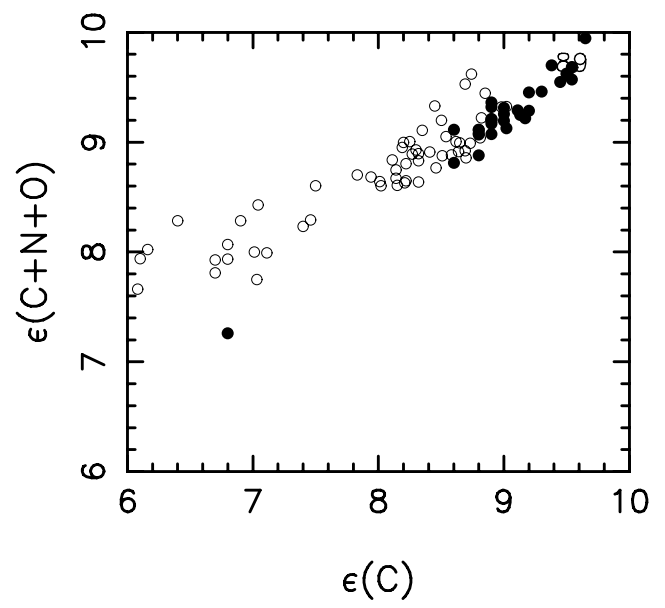

Fig. 4. $\epsilon(\mathrm{C}+\mathrm{N}+\mathrm{O})$ vs. $\epsilon(\mathrm{C})$.

seen so prominently for a class of $\mathrm{PNe}$ as for our $\mathrm{R} \mathrm{CrB}$ and extreme helium stars subsample of post-AGB stars. One interpretation of such an anticorrelation is the production of $\mathrm{N}$ at the expense of $\mathrm{O}(\mathrm{ON}$ cycle) brought to the stellar surface by the 2nd dredge-up. However, the N/O values reached by $\mathrm{R} \mathrm{CrB}$ stars are much higher than for the remaining post-AGB stars and for planetary nebulae. Asplund et al. (2000) argue that CNO cycling on He-burning products has to be invoked to reach this high $\mathrm{N}$ enhancement.

\subsection{Indications of dredge-up}

Figure 4 shows $\epsilon(\mathrm{C}+\mathrm{N}+\mathrm{O})$ vs. $\epsilon(\mathrm{C})$. Only objects in which the abundances of the three elements $(\mathrm{C}, \mathrm{N}$, and $\mathrm{O}$ ) are available are represented here. This plot is very similar to the plot presented by Kingsburgh \& Barlow (1994) and Leisy \& Dennefeld (1996) for planetary nebulae, but with a larger number of points. The objects with the highest carbon abundances, which are mainly $\mathrm{R} \mathrm{CrB}$ stars and extreme helium stars, are carbon dominated. This agrees with a scenario of $\mathrm{C}$ being produced by 

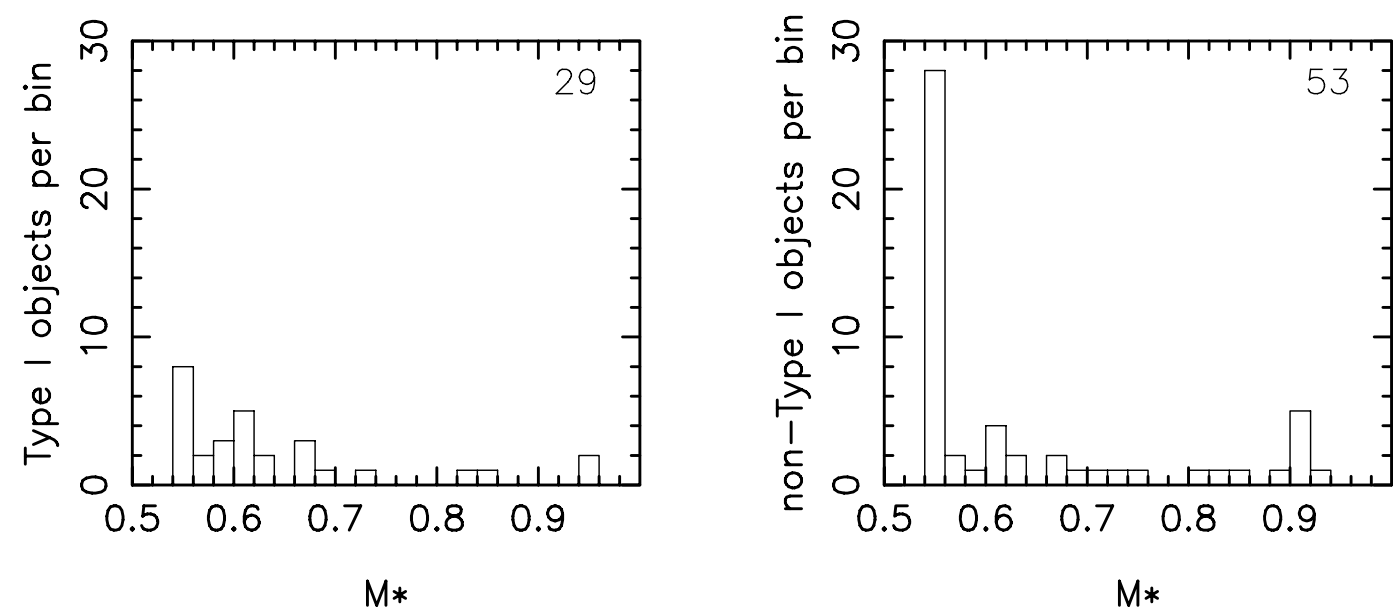

Fig. 5. Stellar mass distribution for type I post-AGB stars (left) and non-type I post-AGB stars (right). We call type I those objects in which N/S is higher than the solar $(\mathrm{C}+\mathrm{N}) / \mathrm{S}$ value.

the triple $\alpha$ reaction and brought to the star surface by the third dredge-up.

\subsection{Dredge-up and stellar mass}

A more quantitative way to define whether dredge-up mechanisms have occurred is to compare abundance ratios observed in post-AGB stars or planetary nebulae with estimates of the initial abundance ratios. For example, Kingsburgh \& Barlow (1994) have proposed to call type I PNe (understood as "objects that have experienced envelope-burning conversion to nitrogen of dredged up primary carbon") those objects in which the nitrogen abundance exceeds its progenitor's $\mathrm{C}+\mathrm{N}$ abundance. As a proxy to the progenitor's $\mathrm{C}+\mathrm{N}$ abundance, they use the value of $\mathrm{C}+\mathrm{N}$ in the Orion nebula. Since the post-AGB considered here are not necessarily all close to the Sun, and because of abundance gradients in the Galaxy, perhaps a safer way is to compare the N/S value in the post-AGB stars to the solar $(\mathrm{C}+\mathrm{N}) / \mathrm{S}$ value, instead of using abundances with respect to hydrogen. For the solar abundances, we rely on the compilation by Lodders (2003). Defining as type I those objects in which $\mathrm{N} / \mathrm{S}$ is larger than the solar $(\mathrm{C}+\mathrm{N}) / \mathrm{S}$ ratio, we show histograms of masses of type I (left) and non-type I (right) post-AGB stars in Fig. 5. It is seen that, while type I objects extend over the entire range of masses in our sample, more than half of the non-type I objects have masses below $0.56 M_{\odot}$. The difference in stellar mass distributions is so tremendous that it is highly significant, even taking into account the fact that error bars on stellar masses are large (as seen in Table 1 and discussed in Sect. 2.1). The conclusion remains the same, although not so strong, if we use $\mathrm{Zn}$ instead of $\mathrm{S}$ as the metallicity indicator. However, when considering only R CrB and extreme helium stars, no such difference is seen.

Similarly, one can identify objects that have experienced 3rd dredge-up as those objects in which $(\mathrm{C}+\mathrm{N}+\mathrm{O}) / \mathrm{S}$ is larger than in the Sun. Figure 6 plots $[(\mathrm{C}+\mathrm{N}+\mathrm{O}) / \mathrm{S}]$ (i.e. the logarithm of $(\mathrm{C}+\mathrm{N}+\mathrm{O}) / \mathrm{S}$ minus the logarithm of the solar value of this ratio) as a function of the stellar mass. This diagram shows that, according to our definition $([(\mathrm{C}+\mathrm{N}+\mathrm{O}) / \mathrm{S}]>0)$, the vast

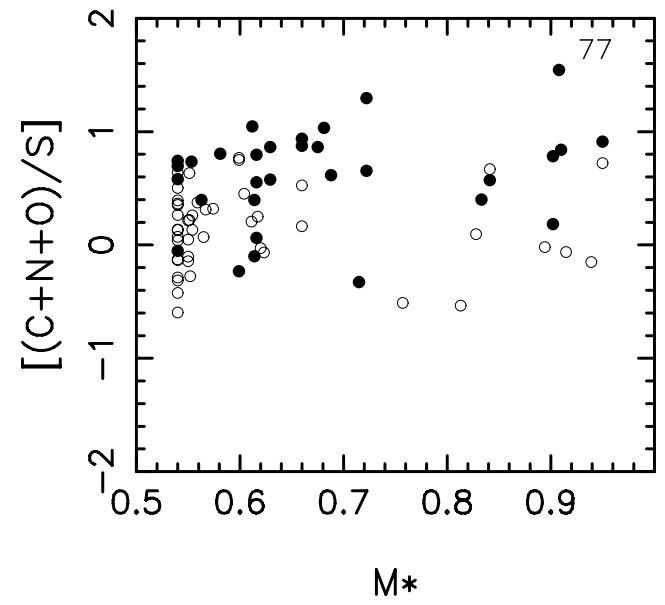

Fig. 6. $[(\mathrm{C}+\mathrm{N}+\mathrm{O}) / \mathrm{S}]$ (i.e. the logarithm of $(\mathrm{C}+\mathrm{N}+\mathrm{O}) / \mathrm{S}$ minus the logarithm of the solar value of this ratio) as a function of the mass of the post-AGB star. Objects with masses lower than $0.55 M_{\odot}$ have been placed at $0.54 M_{\odot}$, objects with masses larger than $0.94 M_{\odot}$ at $0.95 M_{\odot}$,

majority of post-AGB stars in our sample (about 70\%) have experienced 3rd dredge-up. It also suggests that the mass distributions of the two subclasses do not differ significantly.

\subsection{Dredge-up and metallicity}

Theoretical models (e.g. Marigo 2001) predict that 3rd dredgeup is more important at low metallicity. Figure 7 tests this prediction by plotting $[(\mathrm{C}+\mathrm{N}+\mathrm{O}) / \mathrm{S}]$ as a function of $\epsilon(\mathrm{S})$. It shows a net decrease in the efficiency of the 3 rd dredge-up as the metallicity increases, in agreement with the models. This is the first time that the observational evidence for this is so clear. Note that, qualitatively, the same conclusion can be drawn, at least for bona-fide post-AGB stars, when using $\mathrm{Zn}$ instead of $\mathrm{S}$ as a metallicity indicator, as seen in Fig. 8, which plots $[(\mathrm{C}+\mathrm{N}+\mathrm{O}) / \mathrm{S}]$ as a function of $\epsilon(\mathrm{Zn})$. However, $\mathrm{R} \mathrm{CrB}$ and extreme helium stars tend to have higher $[(\mathrm{C}+\mathrm{N}+\mathrm{O}) / \mathrm{S}]$ than bona-fide post-AGB stars of same metallicity. They also behave 


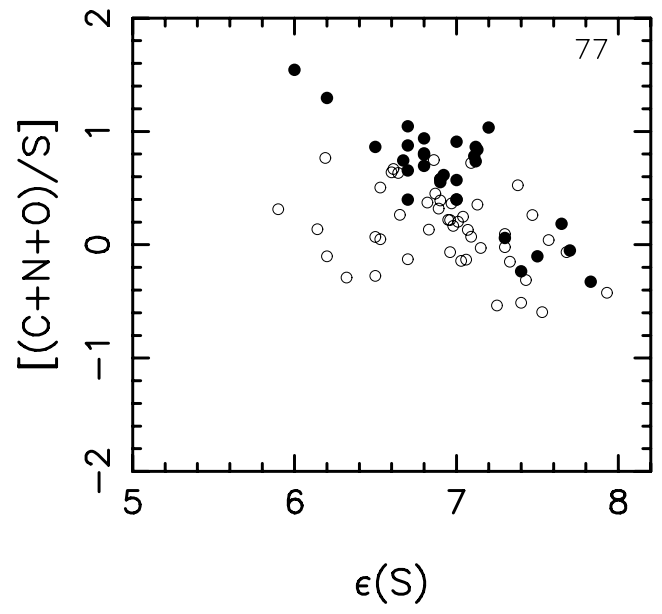

Fig. 7. $[(\mathrm{C}+\mathrm{N}+\mathrm{O}) / \mathrm{S}]$ as a function of the metallicity as measured by $\epsilon(\mathrm{S})$.

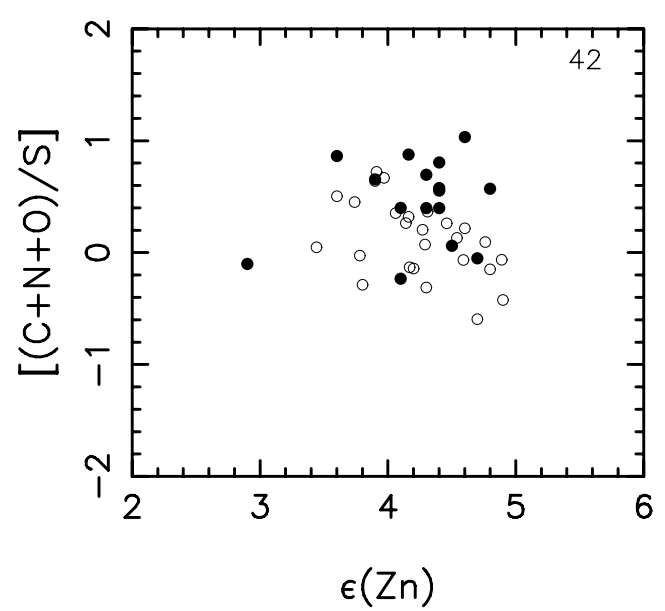

Fig. 8. $[(\mathrm{C}+\mathrm{N}+\mathrm{O}) / \mathrm{S}]$ as a function of the metallicity as measured by $\epsilon(\mathrm{Zn})$.

differently in Figs. 7 and 8, but more Zn abundance determinations would be necessary to make this clear.

\subsection{Carbon-rich versus oxygen-rich post-AGB stars.}

Figure 9 displays the value of $\mathrm{C} / \mathrm{O}$ as a function of stellar mass. It clearly shows that oxygen-rich stars, at least in our sample, tend to accumulate at the lowest masses, while this is not the case for carbon-rich stars. Note also that the proportion of carbon-rich stars is $36 \%$, while the proportion of stars having experienced 3rd dredge-up is as large as about $70 \%$. Of course, these percentages should be taken with a grain of salt since the errors in determined abundances may place an object in the wrong category. However, the difference between these percentages and the large spread of $\mathrm{C} / \mathrm{O}$, as well as $[(\mathrm{C}+\mathrm{N}+\mathrm{O}) / \mathrm{S}]$ values, argues in favour of the number of carbon-rich stars being significantly smaller than the number of stars having experienced 3rd dredge-up. Qualitatively, this is expected, since 3rd dredge-up is not necessarily sufficient to produce a carbon star.

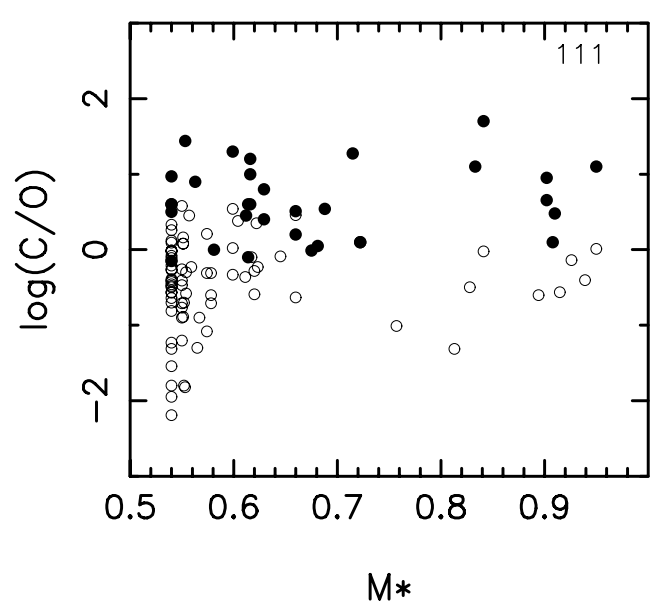

Fig. 9. Photospheric $\mathrm{C} / \mathrm{O}$ versus the mass of the post-AGB stars.

\section{Summary, open questions, and prospects}

The main aim of this paper was to show the utility of post-AGB stars to test theories of AGB nucleosynthesis. So far, the only tests of AGB nucleosynthesis based on large samples have been made using planetary nebulae. Post-AGB stars have several advantages over planetary nebulae: 1) abundances of a large variety of elements can be derived, including of $s$-process elements; 2) the abundance of carbon, an extremely important element for the diagnostics, is known with the same accuracy as the other elements, while in planetary nebulae, the carbon abundance is significantly less reliable and more difficult to obain than that of $\mathrm{O}$ and $\mathrm{N} ; 3$ ) the determination of the atmospheric parameters $T_{\text {eff }}$, and gravity $g$ allows one to estimate the mass of the post-AGB star by comparison with theoretical stellar evolutionary tracks.

Of course, the study of post-AGB stars has its own difficulties. In particular, the abundance analysis is quite difficult and many effects have to be considered in detail (see e.g. Asplund et al. 2000). The lack of suitable lines for reliable analysis is often a problem: i) for cooler objects, the oxygen abundance is hard to derive, since useful lines of oxygen only start to show up at temperatures above $6000 \mathrm{~K}$; ii) nitrogen is often derived from a few red lines, which are known to suffer non-LTE effects; iii) stars that are not enriched in carbon, sometimes have only a few suitable carbon lines.

We considered all those objects from the present version of the catalogue of post-AGB objects (Szczerba et al. 2001; Szczerba et al., in preparation) for which photospheric chemical abundances have been determined. We plotted diagrams based on these abundances, similar to the ones built for planetary nebulae studies. The same trends as for planetary nebulae were found, but in a clearer fashion (e.g. N/O vs. $\epsilon(\mathrm{O})$, revealing the effect of the $\mathrm{ON}$ cycle, or $(\mathrm{C}+\mathrm{N}+\mathrm{O}) / \mathrm{H}$ vs. $\epsilon(\mathrm{C})$ indicating the presence of objects with $\mathrm{C}$ produced by the triple $\alpha$ reaction). This is extremely encouraging and shows the interest of using post-AGB stars to complement planetary nebulae, despite the difficulties in abundance determinations.

Because the post-AGB stars in our sample do not have all the same metallicities, we argued that a better indicator of third dredge-up and/or hot bottom burning is obtained by 
considering the photospheric abundances of $\mathrm{C}, \mathrm{N}, \mathrm{O}$ with respect to a metallicity indicator (and not with respect to $\mathrm{H}$ ). It is therefore these ratios that we compared with the solar ratios. We show that a convenient metallicity indicator is $S$ (Fe cannot be used for post-AGB objects because dust depletion in former stages may have affected its present photospheric abundance). Following the definition of type I planetary nebulae by Kingsburgh \& Barlow (1994) but accounting for the metallicity, we define a class of type I post-AGB stars. We show that non-type I objects are in vast majority of low mass $\left(M_{\star}<0.56 M_{\odot}\right)$. We also show clear evidence that 3rd dredgeup is more efficient at low metallicity.

We have thus demonstrated the potential of post-AGB stars to constrain the models of AGB stars and the predicted yields. The sample of post-AGB stars is likely to grow in the near future, thanks to ASTRO-F, which is much more sensitive than IRAS and should allow the discovery of many infrared-excess stars among which post-AGB stars are found. This will make the use of post-AGB stars even more attractive and powerful. In the present paper, we limited ourselves to only a few elements and to simple interpretations without direct comparison to models. We did not address the question of observational biases, which should be investigated by performing simulations on models. Future studies will address other issues related to AGB nucleosynthesis, such as the production of $s$-process elements, which are more easily done with post-AGB stars than with planetary nebulae.

Acknowledgements. This work was partly supported by grant 2.P03D.017.25 of the Polish State Committee for Scientific Research and by the European Associated Laboratory "Astronomy PolandFrance". G.S. is grateful to Beatriz Barbuy and Corinne Charbonnel for useful comments on a previous version of the paper. Comments by the referee, M. Reyniers are gratefully acknowledged.

\section{References}

Arellano Ferro, A. A., Giridhar, S., \& Mathias, P. 2001, A\&A, 368, 250 (AFGM01)

Asplund, A., Gustafsson, B., Lambert, D. L., \& Rao, N. K. 2000, A\&A, 353, 287 (AGL00)

Blöcker, T. 1995, A\&A, 299, 755

Boothroyd, A. I., \& Sackmann, I.-J. 1999, ApJ, 510, 232

Charbonnel, C. 2002, Ap\&SS, 281, 161

Clayton, G. C. 1996, PASP, 108, 225

Conlon, E. S., Dufton, P. L., Keenan, F. P., \& McCausland, R. J. H. 1991, MNRAS, 248, 820 (CDK91)

Conlon, E. S., Theissen, A., \& Moehler, S. 1993, A\&A, 269, L1 (CTM93)

Conlon, E. S., Dufton, P. L., \& Keenan, F. P. 1994, A\&A, 290, 897 (CDK94)

Decin, L., Van Winckel, H., Waelkens, C., \& Bakker, E. J. 1998, A\&A, 332, 928 (DvWW98)

Drilling, J. S., Jeffery, C. S., \& Heber, U. 1998, A\&A, 329, 1019 (DJH98)

Forestini, M., \& Charbonnel, C. 1997, A\&AS, 123, 241

Fujimoto, M. Y. 1977, PASJ, 29, 331

Gauba, G., \& Parthasarathy, M. 2003, A\&A, 407, 1007 (GP03)

Gautschy, A., \& Saio, H. 1996, ARA\&A, 1 34, 551

Giridhar, S., Rao, N. K., \& Lambert, D. L. 1994, ApJ, 437, 476 (GRL94)
Giridhar, S., Arellano Ferro, A. A., \& Parrao, L. 1997, PASP, 109, 1077 (GAFP97)

Giridhar, S., Lambert, D. L., \& Gonzalez, G. 1998, ApJ, 509, 366 (GLG98)

Giridhar, S., Lambert, D. L., \& Gonzalez, G. 2000, ApJ, 531, 521 (GLG00)

Gonzalez, G., Lambert, D. L., \& Giridhar, S. 1997a, ApJ, 479, 427 (GLG97a)

Gonzalez, G., Lambert, D. L., \& Giridhar, S. 1997b, ApJ, 481, 452 (GLG97b)

Gonzalez, G., \& Wallerstein, G. 1992, MNRAS, 254, 343 (GW92)

Górny, S. K., Stasińska, G., \& Tylenda, R. 1997, A\&A, 318, 256

Groenewegen, M. A. T., \& de Jong, T. 1993, A\&A, 267, 410

Hambly, N. C., Dufton, P. L., Keenan, F. P., \& Lumsden, S. L. 1996, MNRAS, 278, 811 (HDK96)

Harrison, P. M., \& Jeffery, C. S. 1997, A\&A, 323, 177 (HJ97)

Heber, U. 1983, A\&A, 118, 39 (H83)

Henry, R. B. C. 1990, ApJ, 356, 229

Henry, R. B. C., Liebert, J., \& Boroson, T. A. 1989, ApJ, 339, 872

Henry, R. B. C., Kwitter, K. B., \& Bates, J. A. 2000, ApJ, 531, 928

Herwig, F. 2004, in Asymmetrical Planetary Nebulae III: Winds, Structure and the Thunderbird, ed. M. Meixner, J. H. Kastner, B. Balick, \& N. Soker, ASP Conf. Proc., 313, 387

Hrivnak, B. L., \& Reddy, B. 2003, ApJ, 590, 1049 (HR03)

Iben, I. Jr., \& Truran, J. W. 1978, ApJ, 220, 980

Izzard, R. G., Tout, C. A., Karakas, A. I., \& Pols, O. R. 2004, MNRAS, 350,407

Jeffery, C. S. 1993, A\&A, 279, 188 (J93)

Jeffery, C. S., \& Heber, U. 1992, A\&A, 260, 133 (JH92)

Jeffery, C. S., \& Heber, U. 1993, A\&A, 270, 167 (JH93)

Jeffery, C. S., Hanill, P. J., Harrison, P. M., \& Jeffer, S. V. 1998, A\&A, 340, 476 (JHHJ98)

Jeffery, C. S., Hill, P. W., \& Heber, U. 1999, A\&A, 346, 491 (JHH99)

Karakas, A. I., Lattanzio, J. C., \& Pols, O. R. 2002, PASA, 19, 515

Kendall, T. R., Dufton, P. L., Keenan, F. P., Beers, T. C., \& Hambly, N. C. 1997, A\&A, 317, 82 (KDK97)

Kilkenny, D., \& Lydon, J. 1986, MNRAS, 218, 218 (KL86)

Kim, Y.-C., Demarque, P., Yi, S. K., \& Alexander, D. R. 2002, ApJS, 143,499

Kingsburgh, R. L., \& Barlow, M. 1994, MNRAS, 271, 257

Klochkova, V. G. 1995, MNRAS, 272, 710 (K95)

Klochkova, V. G., \& Panchuk, V. E. 1996, Bull. Spec. Astrophys. Observ., 41, 5 (KP96)

Klochkova, V. G., Szczerba, R., Panchuk, V. E., \& Volk, K. 1999, A\&A, 345, 905 (KSP99)

Klochkova, V. G., Szczerba, R., \& Panchuk, V. E. 2000a, Astron. Lett., 26, 88 (KSP00a)

Klochkova, V. G., Szczerba, R., \& Panchuk, V. E. 2000b, Astron. Lett., 26, 439 (KSP00b)

Klochkova, V. G., Yushkin, M. V., Miroshnichenko, A. S., Panchuk, V. E., \& Bjorkman, K. S. 2002a, A\&A, 392, 143 (KYM02)

Klochkova, V. G., Panchuk, V. E., \& Tavolzhanskaya, N. S. 2002b, Astron. Lett., 28, 49 (KPT02)

Kodaira, K. 1973, A\&A, 22, 273

Kwok, S., Volk, K., \& Bidelman, W. P. 1997, ApJS, 112, 557 (KVB97)

Iben, I. Jr., Tutukov, A. V., \& Yungelson, L. R. 1986, ApJ, 456, 750

Jura, M. 1986, ApJ, 309, 732

Lambert, D. L., in the XIII Canary Islands Winter School of Astrophysics, "Cosmochemistry. The melting pot of the elements", ed. C. Esteban, R. J. Garcia-López, A. Herrero, \& F. Sánchez (Cambridge University Press), 81

Lattanzio, J. 2002, New Astron. Rev., 46, 469

Leisy, P., \& Dennefeld, M. 1996, A\&AS, 116, 95 
Lodders, K. 2003, ApJ, 591, 1220

Luck, R. E., \& Bond, H. E. 1984, ApJ, 279, 729 (LB84)

Luck, R. E., Lambert, D. L., \& Bond, H. E. 1983, PASP, 95, 413 (LLB83)

Luck, R. E., Bond, H. E., \& Lambert, D. L. 1990, ApJ, 357, 188 (LBL90)

Maas, T., Van Winckel, H., \& Waelkens, C. 2002, A\&A, 386, 504 (MvWW02)

Maas, T., Van Winckel, H., \& Lloyd Evans, T. 2005, A\&A, 429, 297 (MvWLE05)

Marigo, P. 2001, A\&A, 370, 194

Marigo, P., Bressan, A., \& Chiosi, C. 1996, A\&A, 313, 545

Marigo, P., Bernard-Salas, J., Pottasch, S. R., Tielens, A. G. G. M., \& Wesselius, P. R. 2003, A\&A, 409, 619

Mathis, J. S., \& Lamers, H. J. G. L. M. 1992, A\&A, 259, 39

McCausland, R. J. H., Conlon, E. S., Dufton, P. L., \& Keenan, F. P. 1992, ApJ, 394, 298 (MCD92)

Mishenina, T. V., Kovtyukh, V. V., Soubiran, C., Travaglio, C., \& Busso, M. 2002, A\&A, 396, 189

Moehler, S., \& Heber, U. 1998, A\&A, 335, 985 (MH98)

Mooney, C. J., Rolleston, W. R. J., Keenan, F. P., et al. 2002, MNRAS, 337, 851 (MRK02)

Munn, K. E., Dufton, P. L., Smartt, S. J., \& Hambly, N. C. 2004, A\&A, 419, 713 (MDS04)

Napiwotzki, R., Heber, U., \& Köppen, J. 1994, A\&A, 292, 239 (NHK94)

Norris, J. E., Ryan, S. G., \& Beers, T. C. 2001, ApJ, 561, 1034

Parthasarathy, M., García-Lario, P., Sivarani, T., Manchado, A., \& Sanz Fernanández de Córdoba, L. 2000, A\&A, 357, 241 (PGS00)

Pereira, C. B., Lorenz-Martins, S., \& Machado, M. 2004, A\&A, 422, 637 (PLM04)

Pandey, G., Rao, N. K., Lambert, L. D., Jeffery, C. S., \& Asplund, M. 2001, MNRAS, 324, 937 (PRL01)

Pandey, G., Lambert, L. D., Rao, N. K., \& Jeffery, C. S. 2004, ApJ, 602, L113 (PLRJ04)

Peimbert, M. 1978, in Planetary Nebulae, IAU Symp., 78, 217

Preston, G. W., Krzemi/'nski, W., Smak, J., \& Williams, J. A. 1963, ApJ, 137, 401

Rao, N. K., Giridhar, S., \& Ashoka, B. N. 1990, MNRAS, 244, 29 (RGA90)

Rao, N. K., Goswami, A., \& Lambert, D. L. 2002, MNRAS, 334, 129 (RGL02)

Rao, N. K., \& Lambert, D. L. 2003, PASP, 115, 1304 (RL03)

Reddy, B., \& Hrivnak, B. J. 1999, AJ, 117, 1834 (RH99)

Reddy, B., Parthasarathy, M., \& Sivarani, T. 1996, A\&A, 313, 191 (RPS96)

Reddy, B., Bakker, E. J., \& Hrivnak, B. J. 1999, AJ, 117, 1834 (RBH99)
Reddy, B., Lambert, D. L., Gonzalez, G., \& Yong, D. 2002, ApJ, 564, 482 (RLG02)

Renzini, A. 1979, in Stars and Star Systems, ed. B. E. Westerlund (Dordrecht: Reidel), 155

Renzini, A., \& Voli, M. 1981, A\&A, 94, 175

Reyniers, M., \& Van Winckel, H. 2001, A\&A, 365, 465 (RvW01)

Reyniers, M., Deroo, P., Van Winckel, H., Goriely, S., \& Siess, L. 2004a, Mem. S. A. It., 75, 584 (RDvW04)

Reyniers, M., Van Winckel, H., Gallino, R., \& Straniero, O. 2004b, A\&A, 417, 269 (RvWG04)

Ryans, R. S. I., Dufton, P. L., Mooney, C. J., et al. 2003, A\&A, 401, 1119 (RDM03)

Schönberner, D. 1983, ApJ, 272, 708

Sivarani, T., Parthasarathy, M., García-Lario, P., Manchado, A., \& Pottasch, S. R. 1999, A\&AS, 137, 505 (SPG99)

Stasińska, G. 2004, in the XIII Canary Islands Winter School of Astrophysics, Cosmochemistry. The melting pot of the elements, ed. C. Esteban, R. J. Garcia-López, A. Herrero, \& F. Sánchez (Cambridge University Press), 115

Stasińska, G., Górny, S. K., \& Tylenda, R. 1997, A\&A, 327, 736

Szczerba, R., Górny, S. K., \& Zalfresso-Jundziłło 2001, in PostAGB Objects as a Phase of Stellar Evolution, ed. R. Szczerba, \& S. K. Górny, ASSL, 265, 13

Takeda, Y. 2003, A\&A, 402, 343

Thévenin, F., Parthasarathy, M., \& Jasniewicz, G. 2000, A\&A, 359, 138 (TPJ00)

Van Winckel, H. 1995, Ph.D. Thesis (vW95)

Van Winckel, H. 1997, A\&A, 319, 561 (vW97)

Van Winckel, H., \& Reyniers, M. 2000, A\&A, 354, 135 (vWR00)

Van Winckel, H., Oudmaijer, R. D., \& Trams, N. R. 1996a, A\&A, 312, 553 (vWOT96)

Van Winckel, H., Waelkens, C., \& Waters, L. B. F. M. 1996b, A\&A, 306, L37 (vWWW96)

Vassiliadis, E., \& Wood, P. R. 1994, ApJS, 92, 125

Venn, K. A., Smartt, S. J., Lennon, D. J., \& Dufton, P. L. 1998, A\&A, 334, 987 (VSL98)

Ventura, P., \& D'Antona, F. 2005a, A\&A, 431, 279

Ventura, P., \& D'Antona, F. 2005b [arXiv: astro-ph/0505221]

Waelkens, C., Van Winckel, H., Bogaert, E., \& Trams, N. R. 1991, A\&A, 251, 495 (WvWB91)

Waelkens, C., Van Winckel, H., Trams, N. R., \& Waters, L. B. F. M. 1992, A\&A, 256, L15 (WvWTW92)

Walker, H. J., \& Schönberner, D. 1981, A\&A, 97, 291 (WS81)

Začs, L., Klochkova, V. G., \& Panchuk, V. E. 1995, MNRAS, 275, 764 (ZKP95)

Začs, L., Klochkova, V. G., Panchuk, V. E., \& Spélmanis, R. 1996, MNRAS, 282, 1171 (ZKP96) 